\title{
California's COVID-19 economic shutdown reveals the fingerprint of systemic environmental racism
}

\author{
Richard Bluhm ${ }^{1,2+}$ (bluhm@mak.uni-hannover.de) \\ Pascal Polonik ${ }^{3+}$ (ppolonik@ucsd.edu) \\ Kyle S. Hemes ${ }^{4+}$ (khemes@stanford.edu) \\ Luke C. Sanford ${ }^{2,5+}$ (lcsanford@ucsd.edu) \\ Susanne A. Benz ${ }^{5+}$ (sabenz@ucsd.edu) \\ Morgan C. Levy ${ }^{3,5+}$ (mclevy@ucsd.edu) \\ Katharine Ricke ${ }^{3,5}$ (kricke@ucsd.edu) \\ Jennifer A. Burney ${ }^{5 *}$ (jburney@ucsd.edu) \\ ${ }^{1}$ Leibniz University Hannover, Germany \\ ${ }^{2}$ Department of Political Science, UC San Diego, USA \\ ${ }^{3}$ Scripps Institution of Oceanography, UC San Diego, USA \\ ${ }^{4}$ Stanford Woods Institute for the Environment, Stanford University, USA \\ ${ }^{5}$ School of Global Policy and Strategy, UC San Diego, USA \\ ${ }^{+}$These authors contributed equally. \\ ${ }^{*}$ Communicating author: jburney@ucsd.edu
}

This paper is a preprint submitted to OSF. It is not yet formally under review, and as such, during the submission and review process, future versions of this manuscript may vary slightly. If accepted, the final version of this manuscript will be linked here. We welcome your feedback. 


\title{
California's COVID-19 economic shutdown reveals the fingerprint of systemic environmental racism
}

\author{
Richard Bluhm ${ }^{1,2+}$, Pascal Polonik ${ }^{3+}$, Kyle S. Hemes ${ }^{4+}$, Luke C. Sanford ${ }^{2,5+}$, Susanne A. \\ Benz $^{5+}$, Morgan C. Levy ${ }^{3,5+}$, Katharine Ricke ${ }^{3,5}$, Jennifer A. Burney ${ }^{5 *}$ \\ ${ }^{1}$ Leibniz University Hannover, Germany \\ ${ }^{2}$ Department of Political Science, UC San Diego, USA \\ ${ }^{3}$ Scripps Institution of Oceanography, UC San Diego, USA \\ ${ }^{4}$ Stanford Woods Institute for the Environment, Stanford University, USA \\ ${ }^{5}$ School of Global Policy and Strategy, UC San Diego, USA \\ ${ }^{+}$These authors contributed equally. \\ *Communicating author: jburney@ucsd.edu
}

\begin{abstract}
Racial and ethnic minorities in the United States often experience higher-thanaverage exposures to air pollution. However, the relative contribution of embedded institutional biases to these disparities can be difficult to disentangle from physical environmental drivers, socioeconomic status, and cultural or other factors that are correlated with exposures under status quo conditions. Over the spring and summer of 2020, rapid and sweeping COVID19 shelter-in-place orders around the world created large perturbations to local and regional economic activity that resulted in observable changes in air pollution concentrations, compositions, and distributions. Here, we use the pandemic-related emergency order and subsequent economic slowdown, together with a differences-in-differences approach, to causally estimate pollution exposure disparities in California. Using both public ground-based sensor data and a citizen-science network of monitors for respirable particulate matter $\left(\mathrm{PM}_{2.5}\right)$, along with satellite records of nitrogen dioxide $\left(\mathrm{NO}_{2}\right)$, we show that the initial sheltering-in-place period produced disproportionate air pollution reduction benefits for Asian, Hispanic/Latinx, and lowincome communities. By linking these pollution data with weather, geographic, socioeconomic, and mobility data in difference-in-differences models, we demonstrate that these disparate pollution reductions cannot be explained by environmental conditions, geography, income, or local economic activity and are instead driven by non-local activity. This study thus provides causally-identified evidence of systemic racial and ethnic bias in pollution control under businessas-usual conditions.
\end{abstract}


There exist substantial concerns in the United States about the pervasive harms of racism, which modern scholarship conceptualizes as either active or passive normalization of racial or ethnic inequities (1).* Particularly worrisome is the potential for institutionalized (or systemic) racism in the form of policies, regulations, and norms that favor certain racial or ethnic groups (2) - to perpetuate such harm via democratic processes. Rigorous quantitative evidence of institutional racism can be difficult to come by, because the effects of various social and institutional processes that embed bias (for example urban planning and environmental regulation) often overlap in space and time, and thus stymie attempts at more specific attribution (e.g., (3)). This in turn makes policy proposals that address racism head-on more difficult to justify. This has long been the case with "environmental injustice" or the manifestation of systemic racism in environmental policymaking and enforcement $(4) .^{\dagger}$

Disparities in air pollution exposure provide a clear example of this attribution problem (5-7). Air pollution is linked to a wide range of negative health consequences (8) and is estimated to cause nearly 9 million premature deaths globally per year (9). On average, these health effects are not distributed evenly among different demographic groups (10-13), running counter to the notion that society's environmental burdens should be equally shared $(5-7,14)$. However, despite observable exposure gradients across racial and ethnic groups, causally ascribing such inequities to bias in environmental policy has proven difficult. ${ }^{\ddagger}$ Economic and other social policymaking (e.g., housing, transportation, education) over generations has created the modern geography of who lives where. Over time, myriad physical and social confounds (12) - including variable atmospheric transport processes (15), economic inequalities (11), and neighborhood

*Here we use racism in the modern descriptive sense that does not hinge on the intent of the perpetrator(s): that is, actions and policies that promote race-based inequities are racist, whether or not such an outcome is intended.

${ }^{\dagger}$ The term environmental injustice is often used more broadly to describe disparities across multiple demographic dimensions, including, but not limited to, race and ethnicity. Here, for clarity, we use this more specific definition with a primary focus on racism and racial disparities in environmental policy.

${ }^{\ddagger}$ Here we consider 'environmental policy’ to be the full landscape of policies, laws, statutes, regulations, and enforcement mechanisms governing environmental quality. This definition includes gaps; that is, existing loopholes, lack of regulation, and non-enforcement of rules are also forms of policy. 
demographics - have become correlated with present-day pollution exposures. As such, moving beyond simple observations of disparate but confounded exposures in contemporary cross-sections to causal attribution of environmental injustice thus requires a perturbation to the status quo (16). Such a shock to the policy regime was provided by the initial COVID-19 economic shutdown in California (17).

In early 2020, governments implemented unprecedented policies to limit the public health impacts of the COVID-19 pandemic (18), including stay-at-home orders and travel limitations, with California instituting some of the most aggressive lock-down measures in the U.S. (19). The well-known side-effect of these policies was widespread economic slowdown: businesses closed, factories shuttered, and employees temporarily discontinued their daily commutes (20). Because pollutants like particulate matter $\left(\mathrm{PM}_{2.5}\right)$ and nitrogen dioxide $\left(\mathrm{NO}_{2}\right)$ are produced by transportation, industrial processes, energy production, and agriculture (21), pollutant concentrations tend to track aggregate economic activity (22-24). Thus, the economic slowdown corresponded to reductions in satellite and ground station observations of $\mathrm{NO}_{2}$ and $\mathrm{PM}_{2.5}$ concentrations, particularly in transportation-heavy metropolitan regions (25-27). This period thus provides a baseline for a more equitable distribution of pollutants to which the nonshutdown exposures can be compared.

Here we leverage the shutdowns at the early stages of the COVID-19 pandemic (March-April 2020) as a natural experiment that partially disentangles the confounding underlying legacy of historical social and economic policy from air pollution exposures. Because the COVID-19 shutdowns generated a large local economic shock, the time period provides a clearer look into racial and ethnic disparities in air pollution generation and associated pollution control policies that are obscured within the status-quo California economy. ${ }^{\S}$ We employ established statistical

${ }^{\S}$ California is uniquely well-suited context for this study: it is the fifth largest economy in the world $(28,29)$; it is one of the most racially and ethnically diverse states in the country (30), and one of only a handful in which non-Hispanic whites make up less than half the population(31); it is home to four of the top 20 most populous U.S. cities (32); and despite improvements in air quality in the late 1900s and early 2000s $(33,34)$, several California cities still regularly rank as having the most polluted air in the United 
States(35). Finally, California citizens live under a historically rich tapestry of environmental regulation from the Clean Air Act and its amendments at the federal level to local district level rules - that control air pollution from essentially every source in the state. California also has a long history of environmental activism by and on behalf of disadvantaged communities, which have historically experienced higher pollution exposures (36). As such, it is a favorable location for trying to tease apart environmental racism from the legacy of economic policy and other confounds that might also lead to disparate environmental exposures. 


\section{Results}

We describe our approach to parsing pollution exposure inequities and their local and non-local components in a conceptual schematic (Figures $1 \mathrm{G}$ and $\mathrm{S} 1$ ). Using daily and weekly pollution observations, along with demographic, geographic, and mobility data, we estimate how much race and ethnicity alone explain the changes in air pollution exposures experienced during the COVID-19 shutdown in California. We account for time-varying (e.g., local mobility, weather, seasonality), and relatively static factors (e.g., population density, income, proximity to roads) known to contribute to heterogeneous pollution exposure in different areas.

Our study area and data are summarized in Figure 1. Aerosol $\mathrm{PM}_{2.5}$ measurements are drawn from a network of 826 monitors (106 public monitors from the California Air Resources Board (CARB), and 720 privately-owned PurpleAir monitors; Figure S2A), and cover the period from Jan 1 to April 30, for both 2019 and 2020 (to facilitate comparison across economic conditions at the same time of year). The PurpleAir sensors are low-cost and often privately owned; they have been shown to correlate well with research-grade mass-based sensors, though they tend to have a high bias, which we have corrected before analysis (see Methods). The $\mathrm{PM}_{2.5}$ monitors are located in 746 unique census block groups across California. Satellite-derived tropospheric $\mathrm{NO}_{2}$ (Figure 1B) measurements from the TROPOMI instrument cover all 23212 census block groups of California, but at a weekly time scale, due to the overpass frequency of the Sentinel-5 precursor satellite. Local social, demographic, and geographic characteristics (Figure 1C-F), including income and population shares for race and Hispanic/Latinx ethnicity, are heterogeneously distributed across the state: for example, income tends to be higher in coastal communities and cities, and the southeast and Central Valley regions have higher Hispanic/Latinx population shares. Population shares by race are also somewhat spatially clustered: Asian population share is highest in Los Angeles and the San Francisco Bay Area, but census block groups with larger Black population shares are more widely distributed, especially 
in the southern part of the state. This complex human geography demonstrates the importance of rich measurement networks in addressing questions of environmental justice: the PurpleAir monitors provide a 7 -fold increase in the number of sampled census block groups, although this increase still only represents $3.2 \%$ of all California block groups. (For more detail, please see the supplemental information, which includes a detailed mapping tool ${ }^{\|}$.)

In 2019 (the year prior to the pandemic), without controlling for other sources of heterogeneity, areas with lower income and larger Black and Hispanic/Latinx population shares were exposed to higher-than-average concentrations of both $\mathrm{PM}_{2.5}$ and $\mathrm{NO}_{2}$ than wealthier and white, nonHispanic communities (for $\mathrm{PM}_{2.5}$ this corresponded to: $-1.883 \frac{\mu g}{m^{3}}$ for each unit increase in $\ln$ (median income); $0.065 \frac{\mu g}{m^{3}}$ for each percent increase in share Hispanic/Latinx; $0.052 \frac{\mu g}{m^{3}}$ for each percent increase in share Black; and no significant trend for share Asian. For $\mathrm{NO}_{2}$ this corresponded to: $-8.778 \frac{\mu m o l}{m^{2}}$ for each unit increase in $\ln \left(\right.$ median income); $0.740 \frac{\mu m o l}{m^{2}}$ for each percent increase in share Hispanic/Latinx; $0.809 \frac{\mu m o l}{m^{2}}$ for each percent increase in share Black; and $0.400 \frac{\mu m o l}{m^{2}}$ for each percent increase in share Asian) (Figure S4). Such descriptive air quality differences have long been noted by environmental justice scholars and advocates $(36,39-45)$, but these relationships are confounded by other contributors to variations in pollution exposure (Figure S1). Hence, it is difficult to separate the relationship of these measures with demographic groupings themselves from the social and economic characteristics of these groups, including the policies that generated those groupings (e.g., redlining).(46)

The COVID-19 pandemic temporarily removed a large portion of this confounding economic geography by 'turning off' most economic activity in the state, with some variation in local activity across communities, thus facilitating a glimpse into the relative distribution of locally versus non-locally generated pollutants. Figure 2 shows the depth and dimensions of this natural experiment across the state. The unique response to the spread of COVID-19, including stay-

\footnotetext{
THispanic/Latinx ethnicity is tallied independently of other race information in the U.S., and is therefore not mutually exclusive from race (see Methods; Figure S3).

"https://sabenz.users.earthengine.app/view/covid-ej
} 
at-home orders, precipitate a steep decline in the average fraction of the day that people spent away from their homes (hereafter, mobility), a decline which took a little under two weeks after the state-wide emergency declaration (March 4, 2020) to fully emerge (Figure 2A). Importantly, reductions in time away from home did not occur equally for all state residents. Census block groups with relatively high Hispanic/Latinx population fractions both had higher baseline mobility and much smaller mobility reductions during the shutdown than those with relatively low Hispanic/Latinx populations (Figure 2B). This is likely due the greater designation of essential jobs and economic vulnerability to Hispanic/Latinx populations, relative to nonHispanic/Latinx populations, that precludes being able to work from home (47). This disparity is present, though much less pronounced, for census block groups with high and low Black population shares, and the pre- and post- shutdown differences are reversed for high and low Asian population shares. We account for these different responses in the statistical framework described below.

Using a series of statistical difference-in-differences models (see Methods), we estimate the relative magnitudes of the reductions in $\mathrm{PM}_{2.5}$ and $\mathrm{NO}_{2}$ concentrations before and after the shutdown across different demographic gradients (we show and discuss $\mathrm{PM}_{2.5}$ results in the main text, with $\mathrm{NO}_{2}$ results in the SI, for brevity). The best-fit coefficients for these models, plotted in Figure 3 and Figure S6), correspond to the statistically identifiable expected changes in air pollution, across the COVID-19 shutdown window, for either a 0 vs. $100 \%$ share of a given demographic group at the census block group level or roughly a doubling of non-share variables (e.g., income, road density, population density).** These coefficients show that lowerincome neighborhoods in California experienced greater reductions in $\mathrm{PM}_{2.5}$ concentration (Figure 3A); the positive and statistically significant coefficient for income indicates that lower incomes were strongly associated with more negative shutdown pollutant levels. For example,

${ }^{* *}$ We note that estimating the population average change, on the other hand, would require a stronger statistical assumption than we make about the similarity of other (i.e. seasonality) conditions in 2019 and 2020 (See Methods.) 
our estimates indicate that a block group with an average income that is half that of a wealthier block group would have experienced a $-1 \mu \mathrm{g} / \mathrm{m}^{3}$ greater reduction in $\mathrm{PM}_{2.5}$ exposures. Changes in mobility, road density, and population density at the level of a census block group are only weakly associated with changes in $\mathrm{PM}_{2.5}$ concentrations (Figure $3 \mathrm{~A}$ ).

We consider mobility to be a proxy for local pollution-causing economic activity, and assume that decreased mobility directly corresponded to reduced vehicle emissions along with a suite of local business-related emissions (e.g., restaurants were forced to close). Therefore, the relationship between the relative decline in local mobility and the relative decline in local air pollution gives insight into the pollution impacts of a block group's own economic activity. Figure 3B shows that residents in lower-income neighborhoods reduced their mobility less than richer neighborhoods during the shutdown period. Combined with the fact that lower-income areas experienced a larger drop in $\mathrm{PM}_{2.5}$ concentrations, this finding suggests that local activity is not the primary driver of disparate exposures across the income gradient in California.

To further probe potential heterogeneity in the magnitude of shutdown impacts, we examine exposure changes across neighborhood demographic gradients - with and without accounting for various local characteristics (Figure 3C). We identify substantial racial and ethnic disparities in air quality improvements, even when accounting for income, road and population density, and very fine-grained differences in weather over space and time that strongly affect surface pollutant concentrations (see Methods and Supplement). A ten percentage point (pp) increase in the Hispanic/Latinx population of a census block group is associated with a $-0.28 \mu \mathrm{g} / \mathrm{m}^{3}$ reduction in $\mathrm{PM}_{2.5}$ concentration after the shutdown. This falls to about $-0.24 \mu \mathrm{g} / \mathrm{m}^{3}$ once we include local mobility and allow for heterogeneous effects of the shutdown in terms of income, road density, and population density (mobility impacts are shown directly in Figure 3D). This is evidence of a disproportionate pollution burden experienced by predominantly Hispanic/Latinx communities under business-as-usual conditions - only about a seventh of which is explained by differences in incomes and other location characteristics. We observe a nearly identical change 
of $-0.22 \mu \mathrm{g} / \mathrm{m}^{3}$ in $\mathrm{PM}_{2.5}$ concentration for every ten pp increase in the Asian population of a block group after accounting for heterogeneous effects in location characteristics. Differences in $\mathrm{PM}_{2.5}$ concentration for Black communities are statistically insignificant across all model specifications. This may be due to a smaller Black population share overall (around 7\%) and the fact that there are relatively few majority Black census block groups in California (Figure 1F). These findings are largely consistent with an analogous set of models run using weekly satellitederived $\mathrm{NO}_{2}$ concentrations as the outcome, although some small differences between the two reflect both coverage discrepancies and the distributions of $\mathrm{PM}_{2.5}$ and $\mathrm{NO}_{2}$ sources in the state (Figure S6).

Consistent with other research (48-51), we find large differences in mobility across different income and racial groups. Census block groups with high Hispanic/Latinx and Black populations had smaller mobility reductions during the shutdown than predominantly white neighborhoods (0.8 pp for every $10 \mathrm{pp}$ increase in the population share). However, these differences can be completely accounted for by allowing for heterogeneous effects in income. This suggests that mobility during the pandemic is mainly a function of the economic ability to stay home and the probability of belonging to the essential workforce, rather than other characteristics associated with different neighborhoods. This does not hold for block groups with a greater share of the Asian population. Here we estimate a -0.20 pp decrease in mobility for a 10 pp increase in the census block group Asian population share. The effect falls to - 0.14 pp but remains highly significant even after allowing for heterogeneous responses to other block group characteristics.

Hispanic/Latinx and Asian are the two largest racial and ethnic minority groups in the state, making up about $39 \%$ and $16 \%$ of the population respectively. However, beyond the minority designation, these two groups are very different in their political histories and socioeconomic attributes. Asian Californians are predominately concentrated in urban areas and have on average higher incomes and education, whereas Hispanic/Latinx populations are more skewed towards rural areas, and have on average lower incomes and education.(31) Moreover, as 
described above, the two groups had opposite mobility responses to the shutdown. Despite these large circumstantial differences, we show that their disproportionate exposure to economy-scale pollution is substantially similar, providing strong, albeit indirect, evidence of the influence of systemic racism in the mechanisms and institutions responsible for pollution control.

While our results (Figure 3) show that racial and ethnic disparities in pollutant exposure are robust, and not fully explained by local mobility, income, or other characteristics, a key remaining concern is sampling bias. Ground-based monitors might be placed in a nonrepresentative sub-sample of census block groups, as CARB stations are relatively sparse, and PurpleAir monitors are privately purchased and placed. We find that CARB monitor placement appears to better represent California's disadvantaged communities - these public monitors are more likely to be placed in poorer, more rural, and more racially and ethnically diverse neighborhoods (Figure 4). The PurpleAir network is unsurprisingly slanted towards wealthier locations and under-represents the Hispanic/Latinx population of California.

We therefore derive a set of weightings from these distributions (see Methods and Figure S2B) to combine measurements and better represent statewide demographics in unmeasured census block groups; we use these weightings in our main estimates (i.e. Figure 3). We also re-estimate impacts using several subsets of census block groups, including only CARB monitors, only PurpleAir monitors, and both networks combined with and without weighting (Figure 4). The significant racial disparities calculated from our weighted sample are consistent with most estimates from the unweighted samples (Figure 4). The CARB-only estimates have much larger uncertainties, due in part to the vastly smaller sample size. The bias towards more rural areas in the state's CARB network manifests in larger magnitude estimates for the role of population and road densities in determining air pollution disparities. 


\section{Discussion}

Here we provide new causal estimates of inequality in air pollution exposure and identify a unique fingerprint of systemic racial and ethnic bias in environmental policy in the status quo generation and control of pollutants in the California economy. While this finding is robust to various specifications and data subsets, and consistent across surface- and satellite-based data, our analysis nevertheless requires some contextualization, and care in interpretation. Importantly, while we note that exposure disparities are not explained by local mobility, the ability to fully distinguish local and non-local economic activity is limited; representative spatial scales of local versus non-local may vary (e.g., geographically, culturally, seasonally). We additionally note that while we focus on the non-local drivers of exposure disparities, local mobility-related pollution generation may nevertheless be caused by structurally unjust policy in other sectors (e.g., housing, transportation). More broadly, it is important to recognize that contemporary and historical biases in other policy areas can lead to disparate average exposures, even if environmental policy were unbiased. This may be what explains our finding of higher average pollution exposures, but no disproportionate air quality benefit from the COVID-19 shutdowns for Black communities in California.

We also consistently identified that lower income communities in the state are disproportionately affected by economy-scale pollution. While income is primarily employed as a control in our analysis, this income disparity presents an important environmental justice concern in and of itself, and presents policy challenges that are unique from those associated with combating institutional racism. California has one of the highest rankings for income inequality among U.S. states (31), and our findings provide additional evidence both that wealthier communities are able to both buy environmental quality (i.e., higher housing prices embed air quality) and can afford to stay at home more fully during a pandemic.

Our analysis reveals not only disparities in pollution exposure, but inequality in local air 
pollution information itself. Because air pollution monitoring networks are sparse, researchers have increasingly used Chemical Transport Models (CTMs) to model pollutant exposures and map them to local socio-economic and demographic characteristics. While CTMs have become ever more powerful and accurate, they require accurate emissions inventories as inputs; although rapidly improving for long-lived greenhouse gases (e.g., (52)), such inventories for air pollution, unfortunately, are notoriously uncertain over short time scales and under abnormal economic conditions.(53) Statistical studies like ours also have shortcomings: while they draw on highfrequency data of actual ground-level (or atmospheric column) pollution and thus avoid the need for emissions inventories, they nevertheless require accurate and unbiased characterization of the system under study. While we do not explicitly account for point source emissions or wind in this study, we use areal road density summaries (see Methods), and detailed temperature, precipitation, and relative humidity controls (see Figure S7), which should capture much of this variation. Additionally, because our analysis focuses on differences in the same block group over time, the average influences of these and other unobserved factors are taken into account. Still, we cannot rule out that some of our measured effects may be driven by either variations in emissions or meteorological conditions that are correlated with both the demographic characteristics of a neighborhood and the COVID-related shutdown. Future studies could focus on more thoroughly accounting for natural seasonal swings in air pollution and the full range of its spatial and temporal variability through the inclusion of more years of data.(17) This was not possible here due to the short timescale of PurpleAir data availability. Nevertheless, our use of 2019 as a comparison for 2020, and similar estimates made with pre and post-shutdown 2020 data alone (see Table S1), underscore that the exposure disparities we estimate are not likely to be systematically changed by inclusion of more years of observations.

These findings have important implications for air pollution measurement strategies to address environmental injustice. As we show, monitor placement matters for detection of disparate exposures. CARB recently re-focused air quality monitoring in designated environmental justice 
communities (54), and its monitor placement has resulted in a more accurate sample of the state's Hispanic/Latinx population distribution than (e.g.) PurpleAir. The PurpleAir monitoring network, established through the individual purchase and placement of (relatively) low-cost sensors, shows both that citizen science networks can be exceedingly useful for increasing the amount of public data, but that those networks are unlikely to be optimally placed for addressing environmental justice questions, nor do they accurately reflect the spatial distribution of different sub-populations. PurpleAir sensors also require care in correcting biases compared to monitoringgrade instruments (55). On the public monitoring side, local governments that are responsible for choosing locations of sensors mandated by the Clean Air Act (i.e. CARB) may also strategically place sensors to improve their chances of being in attainment $(56,57)$. This strategic placement reduces the ability of those sensor networks to detect environmental injustice (58) and makes adjustments for sampling bias, like those proposed here, relevant for the larger literature. Lastly, while we show that satellite-based observations can be helpful in understanding the spatial distribution of pollutants that underlies ground-based monitoring network samples, satellite data are spatially coarse compared to the average census block group, and are limited temporally. As such, satellites may not be able to replace ground-based monitoring when high spatial and temporal resolution are required. While a more spatially dense ground measurement network would vastly improve the ability to detect and address environmental injustice, reliability, cost, distribution, and data curation would need to be considered in choosing a scale-up strategy $(59,60)$. Our weighting approach can be used to leverage all available (high quality) ground sensors, no matter which placement mechanism is driving their spatial distribution.

The use of California as a study region makes interpretation of our results more straightforward than might be the case in other regions, or over a larger region. First, California's mild climate and predictable seasonality makes it easier to compare two years of observations than would be the case in more variable climates. Second, the lack of coal and fuel heating oil use in California means that the regional (anthropogenic) aerosol chemistry is relatively simple - California's 
$\mathrm{PM}_{2.5}$ includes primary carbonaceous aerosols produced by transportation, and secondary nitrates produced by transportation and agriculture (61). There are relatively few other primary sources of particulate matter in California compared to other regions, particularly outside of the state's summer-fall wildfire season, which contributes a large organic carbon burden to the region (62). Our study location and timing also mean that satellite-based $\mathrm{NO}_{2}$ observations are more highly correlated with $\mathrm{PM}_{2.5}$ than they would be in other locations, because the same emissions sources contribute to both in the state (predominantly transportation and agriculture). Studies in more complicated climates, and with a more diverse set of aerosol particulate matter (and precursor) emissions will potentially require more sophisticated statistical techniques to address potential unobserved sources of heterogeneity and to assess whether changes in pollution chemical composition differ across population subgroups.

Our analysis demonstrates that the generation and control of pollution from California's economy-at-large disproportionately affect the state's largest racial and ethnic minority communities. Our unconditional finding of disparate shutdown-related decreases for Asian and Hispanic/Latinx communities is evidence of environmental racism, even if it were entirely driven by income differences. However, when we take a more narrow definition of racism and partial out the impacts of many other variables like income, we still find the same or stronger effects. While this complicates their interpretation, it makes clear that these groups are environmentally disadvantaged through environmental policy failures, beyond what we would expect on the basis of income, roads, weather, etc.

This has several implications for potential policy responses. Many state and federal regulatory impact analyses mandate assessment of impacts to disadvantaged and vulnerable groups (e.g., $(63,64))$. While this sets a clear precedent for including analysis of the distribution of regulatory impacts across socioeconomic groups, best practices for quantitative distributional analysis are not codified to the extent of those for (e.g.) benefit-cost analysis typically used in environmental regulation (65). Furthermore, there is no standard assessment criterion for 
justifying interventions to mitigate inequities (e.g., placement of air pollution monitoring that addresses racial disparities) along the lines of the net benefit criterion (the simple objective that regulatory benefits exceed costs). If racial equity is a matter of concern for decision makers, future revisions to regulatory impact analysis mandates should include protocols with provisions for clear, quantitative standards for equity in regulation and analytical practices for evaluating progress. Finally, we note that public policy interventions are considered most effective when targeted to address a known market failure, and many tools of environmental regulation focus on efficiently reducing these failures. However, when systemic bias is driving adverse outcomes, in the absence of amelioration of the unequal representation in political institutions, public policy intervention focused narrowly on addressing market failures may eventually result in reversion to inequality.(66) 


\section{Data and Methods}

\section{Data}

$\mathbf{P M}_{2.5}$ Data: Surface station measurements of particulate matter with diameter smaller than $2.5 \mu \mathrm{m}\left(\mathrm{PM}_{2.5}\right)$ were downloaded from publicly available databases from PurpleAir and the California Air Resources Board (CARB) (Figure S8). We downloaded all outdoor PurpleAir data available (1891 individual stations) for Jan-Apr 2019 and 2020. PurpleAir sensors are relatively inexpensive and are usually privately owned, but much of the data is publicly available. The quality of these data are lower than data from regulatory monitors, but PurpleAir sensors provide unprecedented spatial coverage. Most PurpleAir sensors contain two Particulate Matter Sensor (PMS) 5003 sensors (Plantower, Beijing, China), which measure particle counts in 6 size bins. Counts are converted to $\mathrm{PM}_{2.5}$ using two proprietary conversions, one intended for indoor use and the other for outdoor use; here we use the outdoor conversion as recommended and tested by Tryner et al. (2020) (67). We also average the two sensors (when available) and exclude days when daily $\mathrm{PM}_{2.5}$ measurements within the same unit differ by at least $5 \mu \mathrm{m} \mathrm{m}^{-3}$ and at least $16 \%$ (55). In limited field evaluations, PurpleAir sensors have been shown to have strong correlations with high-quality sensors (67-70)). Tryner et al. also proposed a correction for effects of relative humidity, which we do not apply in part because we consider daily data rather than sub-daily. We do, however, apply an correction developed by the EPA, which tends to slightly over-correct the high bias of the PurpleAir instruments, meaning the presented results from these sensors are conservative (Figure S9) (55).

We downloaded (May 1, 2020) all hourly CARB $\mathrm{PM}_{2.5}$ data in California available for Jan-Apr 2019 and 2020 using CARB's Air Quality and Meteorological Information System (AQMIS), and calculated the daily mean (150 individual stations). Professional instruments and oversight, particularly for calibration, provide higher confidence in the data quality of the CARB sites. However, there are an order of magnitude fewer CARB stations than PurpleAir sensors in 
California, which means studies using the government data are statistically limited by a relatively small sample size. Unlike PurpleAir sensors, CARB sites often offer a wide variety of air pollutant measurements, though we only use hourly $\mathrm{PM}_{2.5}$ aggregated to the daily mean. For both CARB and PurpleAir data, days with mean $\mathrm{PM}_{2.5}$ equal to zero or greater than $500 \mu \mathrm{g}$ $\mathrm{m}^{-3}$ are removed as outliers. Sites for which we remove more than $10 \%$ of data are excluded from the entire analysis. Sites with less than $80 \%$ data coverage during our study period are also excluded. For models that require 2019 and 2020 data, we apply these requirements to both years independently. This quality filtering removed $5.9 \%$ of daily CARB $\mathrm{PM}_{2.5}$ data, and $11.4 \%$ of daily PurpleAir data resulting in data from 1664 individual stations (119 CARB and 1545 Purple Air). However, only 826 of those (106 CARB and 720 PurpleAir) include data for 2019 and 2020 for the pre-shutdown and shutdown period, and were therefore used in our empirical statistical analysis.

$\mathrm{NO}_{2}$ Data: We used the Copernicus Sentinel-5 Precursor TROPOspheric Monitoring Instrument (TROPOMI) Offline tropospheric NO2 column number density (71) for mean $\mathrm{NO}_{2}$ concentrations of the developed areas of each census block group. TROPOMI has a resolution of 0.01 arc degrees. Data were collected for Jan-Apr 2019 and 2020 and only for developed areas based on the U.S. Geological Survey (USGS) National Land Cover Database (NLCD) 2016 (72). For this study, all data was prepared using the Google Earth Engine Python API (73) and formatted as weekly means for each census block group. Weekly means were chosen to counteract the high frequency of missing data, particularly in northern California (Figure S10).

Climate Data: To gather information on temperature, precipitation, and relative humidity we relied on the Gridded Surface Meteorological dataset (GridMet) (74). GridMet provides daily information in a 4-km resolution across the continental USA. For this study, data were aggregated in Google Earth Engine (73) in its original daily frequency for each $\mathrm{PM}_{2.5}$ measurement station, and as a weekly mean for the $\mathrm{NO}_{2}$-Data for each census block 
group. The weekly mean data was only collected for developed areas based on the U.S.

Geological Survey (USGS) National Land Cover Database (NLCD) 2016 (72).

Mobility Data: We use SafeGraph's Social Distancing Metrics, which were made available for research as part of the company's COVID-19 response. SafeGraph collects and cleans GPS pings from about 45 million mobile devices. The data are available daily at census block group resolution and are close to a random sample of the population. Our primary measure of mobility, not social distancing, is the percent of time spent away from home. We calculate this measure based on the median time (in minutes) that a device was observed at its geohash-7 (about $153 m \times 153 m$ ) home location, which SafeGraph determines as the night time residence of the device in the 6 -weeks prior. The data cover the entire period of observation from Jan 1, 2019 until the end of April 2020.

Demographic Data: We downloaded census block groups level demographic information from the U.S. Census Bureau 2018 5-year American Community Survey (ACS) for all CBGs in California using the tidycensus package (75) for the $\mathrm{R}$ programming environment (76) (June 29, 2020). Demographic features included ACS sample-based CBG-level estimates of: population count; white race count (alone or in combination with one or more other races), or "white"; Black or African American race count (alone or in combination with one or more other races), or "Black"; Asian race count (alone or in combination with one or more other races), or "Asian"; Hispanic or Latino origin (of any race) count, or "Hispanic/Latinx"; and median income. Other race and ethnic groups represent a substantially lower share of the California population, and were therefore excluded from our analysis due to small sample sizes. The CBGlevel "share" of these groups was calculated by dividing the CBG count by the CBG population. Population density was calculated as the CBG population divided by the area of the CBG. Because Hispanic/Latinx is a separate designation from race in the ACS (i.e., those categorized as Hispanic/Latinx may also be of any race), we evaluated how distinct Hispanic/Latinx was from race variables of interest (Figure S3). On average, less than $1 \%$ of those identified at the 
CBG level as Hispanic/Latinx were also identified as Black or Asian; 61\% of Hispanic/Latinx were White. Thus, Hispanic/Latinx is effectively distinct from Asian and Black categorizations, and we consider Hispanic/Latinx, Asian, and Black designations to be unique demographic indicators in our model.

Geographic Data: We calculated road density $\left(\mathrm{m} / \mathrm{km}^{2}\right)$ using The Global Roads Inventory Project (GRIP4) (77) vector dataset for North America, downloaded at https://www.globio.info/download-grip-dataset (April 4, 2020). The GRIP4 dataset harmonizes global geospatial datasets on road infrastructure, including road features that can be categorized as highways, primary roads, secondary roads, tertiary roads and local roads. It is consistent with primary and secondary road classifications from the U.S. Census TIGER/Line shapefiles for roads. To calculate road density for each CBG, we summed road lengths within the area of the $\mathrm{CBG}$, and divided by the area of the CBG. Calculations were done using the $s f$ package (78) in the $\mathrm{R}$ programming environment (76).

\section{Methods}

Study period: We consider three periods between Jan 1 and April 30 in 2019 and 2020. The first period is "pre-shutdown," followed by a "transition," and then "shutdown." The transition is defined as the period between the state-wide emergency declaration (March 4, 2020) and the state-wide stay-at-home order (March 19, 2020). The mobility data demonstrate that activity declined throughout this period (Figure 2). This is consistent with recent literature which shows that fear was a potent driver of the decline in mobility and often preempted county-wide legal restrictions (79). The shutdown period begins with the stay-at-home order and ends at the end of our study period. We exclude the transition from the model analyses described below.

Empirical Strategy: In our statistical analyses, our main dependent variable is an (average) measure of air quality $\left(\mathrm{PM}_{2.5}\right.$ or $\left.\mathrm{NO}_{2}\right)$ in census block group $i$ at day (or week) $t$. We focus 
on block groups to minimize the influence of aggregation bias or the "ecological fallacy" (80) and study temporal variation in air quality across block groups using a differences-indifferences design. Differences-in-differences methods are commonly used to study causal effects in economics (81). Our objective is to estimate the heterogeneity in the effect of the shutdown across different communities, rather than the overall effect of the shutdown. We focus on the racial composition of California's three biggest racial and ethnic minority groups (Hispanic/Latinx, Asian,and Black) to first establish the existence of an air pollution exposure inequity and then include observed characteristics of minority populations to document the racial inequities that remain after accounting for differences in mobility, income and location (82).

A key concern is that differences in air quality are driven by interannual cycles in pollution and particle concentration unrelated to the shutdown. We address this issue in several ways. First, we subtract observed air quality in 2019 from the 2020 value. All annual differences, after aligning the weekdays, are denoted by $\tilde{y}_{i t}=y_{i t}-y_{i, t-364}$. Second, we flexibly control for local weather conditions in 2020 and 2019. Finally, we allow for a rich set of day or week fixed-effects which capture the remaining differences in synoptic scale weather patterns. We estimate the heterogeneous effect of the shutdown using variants of the following specification

$\tilde{y}_{i t}=\sum_{k=1}^{K} \gamma_{k}\left(d_{t} \times x_{i}^{k}\right)+\theta \tilde{M}_{i t}+f^{2020}(T, R H, P)_{i t}+f^{2019}(T, R H, P)_{i t}+\lambda_{t}+\mu_{i}+e_{i t}$

where $d_{t}$ is an indicator for the post-shutdown period, $x_{i}^{k}$ are the population shares of the three minority groups or other time-invariant location characteristics at the census block group level, $\tilde{M}_{i t}$ is the annual difference in mobility, $f^{2020}(\cdot)_{i t}$ and $f^{2019}(\cdot)_{i t}$ approximate the non-linear response of pollution and particle concentration to weather with interacted fixed effects for each decile of temperature, relative humidity and precipitation in the corresponding year, $\lambda_{t}$ are day (or week) fixed effects, $\mu_{i}$ are census block group fixed effects (capturing changes in the number 
of stations in a block group across years), and $e_{i t}$ is an error term. We cluster all standard errors on the county level, as stay-at-home and local health ordinances are spatially and temporally correlated at this level.

We are interested in $\gamma_{k}$ which captures the heterogeneous impact of the shutdown across different demographic gradients (see SI text for a derivation). The baseline effect of the shutdown, $d_{t}$, is not statistically identified without the assumption constant seasonal emissions patterns, as that baseline effect occurs simultaneously for all block groups in California and is therefore collinear with seasonal shifts in air quality that are unrelated to the COVID-19 shutdowns. Heterogeneous impacts are identified by variation among block groups experiencing a COVID-19 shutdown-related air pollution change only, and can be interpreted as the effect of the shutdown relative to some baseline. This requires a weaker assumption: that the inter-annual differences in pollution are not simultaneously correlated with the timing of the shutdown and the spatial distribution of race and income. Our weather controls make this a plausible assumption by accounting for systematic differences in temperature, humidity, and rainfall across different parts of the state. We interpret the coefficient on $d_{t} \times \%$ Hispanic/Latinx as the difference in $\mathrm{PM}_{2.5}$ concentration for a block group which is $100 \%$ Hispanic/Latinx, relative to a block group which is $0 \%$ Hispanic/Latinx. Differences in air pollution concentrations across the shutdown window are typically reductions in air pollution, which we consider to be equivalent to the expected increase after a return to "business-as-usual" conditions.

Software: All data processing and analysis other than acquisition, and pre-processing of mobility information was done using the R programming environment (76) and the python API for Google Earth Engine (73). 


\section{Acknowledgments}

S.A.B. is supported by the Big Pixel Initiative at UC San Diego, J.A.B., M.C.L., and S.A.B. are supported by NSF/USDA NIFA INFEWS T1 \#1619318; J.A.B. and P.P. are supported by NSF CNH-L \#1715557. R.B. is supported by the Alexander von Humboldt Foundation. K.S.H is supported by the Stanford Woods Institute for the Environment.

\section{Author Contributions}

K.S.H., P.P., J.A.B., M.C.L, S.A.B., L.C.S., R.B. designed study. S.A.B., M.C.L., K.S.H., P.P., and R.B. prepared data and code for analyses; R.B. and L.C.S. ran statistical models. All authors interpreted results, prepared figures, wrote, and edited the manuscript together.

\section{References}

[1] Ibram X Kendi. How to be an antiracist. One world, 2019.

[2] Joe Feagin. Systemic racism: A theory of oppression. Routledge, 2013.

[3] Nancy Krieger, Gretchen Van Wye, Mary Huynh, Pamela D Waterman, Gil Maduro, Wenhui Li, R Charon Gwynn, Oxiris Barbot, and Mary T Bassett. Structural racism, historical redlining, and risk of preterm birth in new york city, 2013-2017. American Journal of Public Health, (0):e1-e8, 2020.

[4] David N Pellow. Environmental inequality formation: Toward a theory of environmental injustice. American behavioral scientist, 43(4):581-601, 2000.

[5] Robert D Bullard. The legacy of american apartheid and environmental racism. . John's J. Legal Comment., 9:445, 1993.

[6] Robert J. Brulle and David N. Pellow. Environmental Justice: Human Health and Environmental Inequalities. Annual Review of Public Health, 27(1):103-124, 2006.

[7] Spencer Banzhaf, Lala Ma, and Christopher Timmins. Environmental justice: The economics of race, place, and pollution. Journal of Economic Perspectives, 33(1):185-208, February 2019. 
[8] Gerard Hoek, Ranjini M. Krishnan, Rob Beelen, Annette Peters, Bart Ostro, Bert Brunekreef, and Joel D. Kaufman. Long-term air pollution exposure and cardio- respiratory mortality: a review. Environmental Health, 12(1):43, May 2013.

[9] Richard Burnett, Hong Chen, Mieczysław Szyszkowicz, Neal Fann, Bryan Hubbell, C Arden Pope, Joshua S Apte, Michael Brauer, Aaron Cohen, Scott Weichenthal, et al. Global estimates of mortality associated with long-term exposure to outdoor fine particulate matter. Proceedings of the National Academy of Sciences, 115(38):9592-9597, 2018.

[10] Michael Jerrett. Global Geographies of Injustice in Traffic-Related Air Pollution Exposure. Epidemiology, 20(2):231-233, March 2009.

[11] Anthony Nardone, Joan A. Casey, Rachel Morello-Frosch, Mahasin Mujahid, John R. Balmes, and Neeta Thakur. Associations between historical residential redlining and current age-adjusted rates of emergency department visits due to asthma across eight cities in California: an ecological study. The Lancet Planetary Health, 4(1):e24-e31, January 2020.

[12] Christopher W. Tessum, Joshua S. Apte, Andrew L. Goodkind, Nicholas Z. Muller, Kimberley A. Mullins, David A. Paolella, Stephen Polasky, Nathaniel P. Springer, Sumil K. Thakrar, Julian D. Marshall, and Jason D. Hill. Inequity in consumption of goods and services adds to racial-ethnic disparities in air pollution exposure. Proceedings of the National Academy of Sciences, 116(13):6001-6006, March 2019.

[13] Ihab Mikati, Adam F. Benson, Thomas J. Luben, Jason D. Sacks, and Jennifer RichmondBryant. Disparities in Distribution of Particulate Matter Emission Sources by Race and Poverty Status. American Journal of Public Health, 108(4):480-485, April 2018.

[14] U.S. Environmental Protection Agency. Environmental Justice, November 2014.

[15] Nelson L Seaman. Meteorological modeling for air-quality assessments. Atmospheric environment, 34(12-14):2231-2259, 2000.

[16] Kenneth Y. Chay and Michael Greenstone. The Impact of Air Pollution on Infant Mortality: Evidence from Geographic Variation in Pollution Shocks Induced by a Recession. The Quarterly Journal of Economics, 118(3):1121-1167, August 2003.

[17] Noah S Diffenbaugh, Christopher B Field, Eric A Appel, Ines L Azevedo, Dennis D Baldocchi, Marshall Burke, Jennifer A Burney, Philippe Ciais, Steven J Davis, Arlene M Fiore, et al. The covid-19 lockdowns: a window into the earth system. Nature Reviews Earth \& Environment, pages 1-12, 2020.

[18] Solomon Hsiang, Daniel Allen, Sébastien Annan-Phan, Kendon Bell, Ian Bolliger, Trinetta Chong, Hannah Druckenmiller, Luna Yue Huang, Andrew Hultgren, Emma Krasovich, et al. 
The effect of large-scale anti-contagion policies on the COVID-19 pandemic. Nature, pages $1-9,2020$.

[19] State of California. Stay home Q\&A, August 2020.

[20] Corinne Le Quéré, Robert B. Jackson, Matthew W. Jones, Adam J. P. Smith, Sam Abernethy, Robbie M. Andrew, Anthony J. De-Gol, David R. Willis, Yuli Shan, Josep G. Canadell, Pierre Friedlingstein, Felix Creutzig, and Glen P. Peters. Temporary reduction in daily global CO 2 emissions during the COVID-19 forced confinement. Nature Climate Change, 10(7):647-653, July 2020.

[21] Jing Cai, Yihui Ge, Huichu Li, Changyuan Yang, Cong Liu, Xia Meng, Weidong Wang, Can Niu, Lena Kan, Tamara Schikowski, et al. Application of land use regression to assess exposure and identify potential sources in pm2. 5, bc, no2 concentrations. Atmospheric Environment, 223:117267, 2020.

[22] Mary E. Davis. Recessions and Health: The Impact of Economic Trends on Air Pollution in California. American Journal of Public Health, 102(10):1951-1956, August 2012.

[23] Bryan N Duncan, Lok N Lamsal, Anne M Thompson, Yasuko Yoshida, Zifeng Lu, David G Streets, Margaret M Hurwitz, and Kenneth E Pickering. A space-based, high-resolution view of notable changes in urban nox pollution around the world (2005-2014). Journal of Geophysical Research: Atmospheres, 121(2):976-996, 2016.

[24] Sumil K. Thakrar, Srinidhi Balasubramanian, Peter J. Adams, Inês M. L. Azevedo, Nicholas Z. Muller, Spyros N. Pandis, Stephen Polasky, C. Arden Pope, Allen L. Robinson, Joshua S. Apte, Christopher W. Tessum, Julian D. Marshall, and Jason D. Hill. Reducing Mortality from Air Pollution in the United States by Targeting Specific Emission Sources. Environmental Science $\&$ Technology Letters, July 2020. Publisher: American Chemical Society.

[25] Zander S. Venter, Kristin Aunan, Sourangsu Chowdhury, and Jos Lelieveld. COVID-19 lockdowns cause global air pollution declines. Proceedings of the National Academy of Sciences, July 2020.

[26] M Bauwens, S Compernolle, T Stavrakou, J-F Müller, J Van Gent, H Eskes, Pieternel Felicitas Levelt, R van der A, JP Veefkind, J Vlietinck, et al. Impact of coronavirus outbreak on no2 pollution assessed using tropomi and omi observations. Geophysical Research Letters, 47(11):e2020GL087978, 2020.

[27] Fei Liu, Aaron Page, Sarah A. Strode, Yasuko Yoshida, Sungyeon Choi, Bo Zheng, Lok N. Lamsal, Can Li, Nickolay A. Krotkov, Henk Eskes, Ronald van der A, Pepijn Veefkind, 
Pieternel F. Levelt, Oliver P. Hauser, and Joanna Joiner. Abrupt decline in tropospheric nitrogen dioxide over China after the outbreak of COVID-19. Science Advances, 6(28):eabc2992, July 2020.

[28] U.S. Bureau of Economic Analysis (BEA). Gross Domestic Product by State: 4th Quarter and Annual 2019.

[29] International Monetary Fund. Report for Selected Countries and Subjects, October 2019.

[30] U.S. News and World Reports. Majority of U.S. Cities are Becoming More Diverse, New Analysis Shows, January 2020.

[31] US Census Bureau. ACS Provides New State and Local Income, Poverty and Health Insurance Statistics, September 2019.

[32] US Census Bureau. City and Town Population Totals: 2010-2019, May 2020.

[33] David D Parrish, Jin Xu, Bart Croes, and Min Shao. Air quality improvement in los angeles - perspectives for developing cities. Frontiers of Environmental Science \& Engineering, 10(5):11, 2016.

[34] Erika Garcia, Robert Urman, Kiros Berhane, Rob McConnell, and Frank Gilliland. Effects of policy-driven hypothetical air pollutant interventions on childhood asthma incidence in southern california. Proceedings of the National Academy of Sciences, 116(32):15883-15888, 2019 .

[35] American Lung Association. State of the Air 2020. Technical report, American Lung Association, Chicago, Illinois, 2020.

[36] Manuel Pastor Jr, Rachel Morello-Frosch, and James L Sadd. The air is always cleaner on the other side: Race, space, and ambient air toxics exposures in california. Journal of urban affairs, 27(2):127-148, 2005.

[37] Joakim A. Weill, Matthieu Stigler, Olivier Deschenes, and Michael R. Springborn. Social distancing responses to COVID-19 emergency declarations strongly differentiated by income. Proceedings of the National Academy of Sciences, July 2020.

[38] Giovanni Bonaccorsi, Francesco Pierri, Matteo Cinelli, Andrea Flori, Alessandro Galeazzi, Francesco Porcelli, Ana Lucia Schmidt, Carlo Michele Valensise, Antonio Scala, Walter Quattrociocchi, and Fabio Pammolli. Economic and social consequences of human mobility restrictions under COVID-19. Proceedings of the National Academy of Sciences, 117(27), July 2020. 
[39] Susan A Perlin, R Woodrow Setzer, John Creason, and Ken Sexton. Distribution of industrial air emissions by income and race in the united states: an approach using the toxic release inventory. Environmental Science \& Technology, 29(1):69-80, 1995.

[40] Susan A Perlin, Ken Sexton, and David WS Wong. An examination of race and poverty for populations living near industrial sources of air pollution. Journal of Exposure Analysis \& Environmental Epidemiology, 9(1), 1999.

[41] R Charon Gwynn and George D Thurston. The burden of air pollution: impacts among racial minorities. Environmental health perspectives, 109(suppl 4):501-506, 2001.

[42] Julian D Marshall. Environmental inequality: air pollution exposures in california's south coast air basin. Atmospheric Environment, 42(21):5499-5503, 2008.

[43] Nam P Nguyen and Julian D Marshall. Impact, efficiency, inequality, and injustice of urban air pollution: variability by emission location. Environmental Research Letters, 13(2):024002, 2018.

[44] Lara P Clark, Dylan B Millet, and Julian D Marshall. National patterns in environmental injustice and inequality: outdoor no 2 air pollution in the united states. PloS one, 9(4):e94431, 2014.

[45] Lara P Clark, Dylan B Millet, and Julian D Marshall. Changes in transportation-related air pollution exposures by race-ethnicity and socioeconomic status: Outdoor nitrogen dioxide in the united states in 2000 and 2010. Environmental health perspectives, 125(9):097012, 2017.

[46] Anthony Nardone, Joan A Casey, Rachel Morello-Frosch, Mahasin Mujahid, John R Balmes, and Neeta Thakur. Associations between historical residential redlining and current ageadjusted rates of emergency department visits due to asthma across eight cities in california: an ecological study. The Lancet Planetary Health, 4(1):e24-e31, 2020.

[47] Misael Galdamez, Charlotte Kesteven, and Aaron Melaas. In a vulnerable state.

[48] Giovanni Bonaccorsi, Francesco Pierri, Matteo Cinelli, Andrea Flori, Alessandro Galeazzi, Francesco Porcelli, Ana Lucia Schmidt, Carlo Michele Valensise, Antonio Scala, Walter Quattrociocchi, et al. Economic and social consequences of human mobility restrictions under covid-19. Proceedings of the National Academy of Sciences, 117(27):15530-15535, 2020 .

[49] Michael S Warren and Samuel W Skillman. Mobility changes in response to covid-19. arXiv preprint arXiv:2003.14228, 2020. 
[50] Caroline O Buckee, Satchit Balsari, Jennifer Chan, Mercè Crosas, Francesca Dominici, Urs Gasser, Yonatan H Grad, Bryan Grenfell, M Elizabeth Halloran, Moritz UG Kraemer, et al. Aggregated mobility data could help fight covid-19. Science (New York, NY), 368(6487):145, 2020 .

[51] Serina Chang, Emma Pierson, Pang Wei Koh, Jaline Gerardin, Beth Redbird, David Grusky, and Jure Leskovec. Mobility network models of covid-19 explain inequities and inform reopening. Nature, pages 1-6, 2020.

[52] Zhu Liu, Philippe Ciais, Zhu Deng, Ruixue Lei, Steven J Davis, Sha Feng, Bo Zheng, Duo Cui, Xinyu Dou, Biqing Zhu, et al. Near-real-time monitoring of global co 2 emissions reveals the effects of the covid-19 pandemic. Nature communications, 11(1):1-12, 2020.

[53] Rachel M Hoesly, Steven J Smith, Leyang Feng, Zbigniew Klimont, Greet JanssensMaenhout, Tyler Pitkanen, Jonathan J Seibert, Linh Vu, Robert J Andres, Ryan M Bolt, et al. Historical (1750-2014) anthropogenic emissions of reactive gases and aerosols from the community emission data system (ceds). Geoscientific Model Development, 11:369-408, 2018.

[54] California State Assembly. Ab-617: Nonvehicular air pollution: criteria air pollutants and toxic air contaminants. 2017.

[55] K. Johnson, B. Gantt, I. VonWald, and A. Clements. Pm 2.5 performance across the u.s. 2019 .

[56] Luke Fowler. Local Governments: The "Hidden Partners" of Air Quality Management. State and Local Government Review, 48(3):175-188, September 2016.

[57] Nicholas Z. Muller and Paul A. Ruud. What Forces Dictate the Design of Pollution Monitoring Networks? Environmental Modeling \& Assessment, 23(1):1-14, February 2018.

[58] Corbett Grainger and Andrew Schreiber. Discrimination in Ambient Air Pollution Monitoring? AEA Papers and Proceedings, 109:277-282, May 2019.

[59] Dorothy L Robinson. Accurate, low cost pm2. 5 measurements demonstrate the large spatial variation in wood smoke pollution in regional australia and improve modeling and estimates of health costs. Atmosphere, 11(8):856, 2020.

[60] Thomas Becnel, Kyle Tingey, Jonathan Whitaker, Tofigh Sayahi, Katrina Lê, Pascal Goffin, Anthony Butterfield, Kerry Kelly, and Pierre-Emmanuel Gaillardon. A distributed low-cost pollution monitoring platform. IEEE Internet of Things Journal, 6(6):10738-10748, 2019.

[61] S. Hasheminassab, N. Daher, A. Saffari, D. Wang, B. D. Ostro, and C. Sioutas. Spatial and temporal variability of sources of ambient fine particulate matter $\left(\mathrm{pm}_{2.5}\right)$ in california. Atmospheric Chemistry and Physics, 14(22):12085-12097, 2014. 
[62] Dan Jaffe, William Hafner, Duli Chand, Anthony Westerling, and Dominick Spracklen. Interannual variations in pm2. 5 due to wildfires in the western united states. Environmental science \& technology, 42(8):2812-2818, 2008.

[63] Executive Order 12866: Regulatory Planning and Review, volume 58. 1993.

[64] Executive Order 12898: Federal actions to address environmental justice in minority populations and low-income populations, volume 59. 1994.

[65] Office of Management and Budget. Circular a-4: Regulatory analysis. 2003.

[66] Tseming Yang. Melding civil rights and environmentalism: Finding environmental justice's place in environmental regulation. Harvard Environmental Law Review, 26:1, 2002.

[67] Jessica Tryner, Christian L'Orange, John Mehaffy, Daniel Miller-Lionberg, Josephine C Hofstetter, Ander Wilson, and John Volckens. Laboratory evaluation of low-cost purpleair pm monitors and in-field correction using co-located portable filter samplers. Atmospheric Environment, 220:117067, 2020.

[68] T Sayahi, A Butterfield, and KE Kelly. Long-term field evaluation of the plantower pms low-cost particulate matter sensors. Environmental Pollution, 245:932-940, 2019.

[69] Jianzhao Bi, Avani Wildani, Howard H Chang, and Yang Liu. Incorporating low-cost sensor measurements into high-resolution pm2. 5 modeling at a large spatial scale. Environmental Science $\&$ Technology, 54(4):2152-2162, 2020.

[70] Iasonas Stavroulas, Georgios Grivas, Panagiotis Michalopoulos, Eleni Liakakou, Aikaterini Bougiatioti, Panayiotis Kalkavouras, Kyriaki Maria Fameli, Nikolaos Hatzianastassiou, Nikolaos Mihalopoulos, and Evangelos Gerasopoulos. Field evaluation of low-cost pm sensors (purple air pa-ii) under variable urban air quality conditions, in greece. Atmosphere, 11(9):926, 2020.

[71] J.P. Veefkind, I. Aben, K. McMullan, H. Förster, J. de Vries, G. Otter, J. Claas, H.J. Eskes, J.F. de Haan, Q. Kleipool, M. van Weele, O. Hasekamp, R. Hoogeveen, J. Landgraf, R. Snel, P. Tol, P. Ingmann, R. Voors, B. Kruizinga, R. Vink, H. Visser, and P.F. Levelt. TROPOMI on the ESA sentinel-5 precursor: A GMES mission for global observations of the atmospheric composition for climate, air quality and ozone layer applications. Remote Sensing of Environment, 120:70-83, May 2012.

[72] Limin Yang, Suming Jin, Patrick Danielson, Collin Homer, Leila Gass, Stacie M. Bender, Adam Case, Catherine Costello, Jon Dewitz, Joyce Fry, Michelle Funk, Brian Granneman, Greg C. Liknes, Matthew Rigge, and George Xian. A new generation of the united states national land cover database: Requirements, research priorities, design, and implementation 
strategies. ISPRS Journal of Photogrammetry and Remote Sensing, 146:108-123, December 2018 .

[73] Noel Gorelick, Matt Hancher, Mike Dixon, Simon Ilyushchenko, David Thau, and Rebecca Moore. Google earth engine: Planetary-scale geospatial analysis for everyone. Remote Sensing of Environment, 2017.

[74] John T. Abatzoglou. Development of gridded surface meteorological data for ecological applications and modelling. International Journal of Climatology, 33(1):121-131, December 2011.

[75] Kyle Walker. tidycensus: Load US Census Boundary and Attribute Data as 'tidyverse' and 'sf'-Ready Data Frames, 2020. R package version 0.9.9.5.

[76] R Core Team. R: A Language and Environment for Statistical Computing. R Foundation for Statistical Computing, Vienna, Austria, 2020.

[77] Johan R. Meijer, Mark A. J. Huijbregts, Kees C. G. J. Schotten, and Aafke M. Schipper. Global patterns of current and future road infrastructure. Environmental Research Letters, 13(6):064006, May 2018.

[78] Edzer Pebesma. Simple Features for R: Standardized Support for Spatial Vector Data. The R Journal, 10(1):439-446, 2018.

[79] Austan Goolsbee and Chad Syverson. Fear, lockdown, and diversion: Comparing drivers of pandemic economic decline 2020. Working Paper 27432, National Bureau of Economic Research, June 2020.

[80] Brett M. Baden, Douglas S. Noonan, and Rama Mohana R. Turaga. Scales of justice: Is there a geographic bias in environmental equity analysis? Journal of Environmental Planning and Management, 50(2):163-185, 2007.

[81] Jeffrey M. Wooldridge. Asymptotic properties of weighted m-estimators for variable probability samples. Econometrica, 67(6):1385-1406, 1999.

[82] Spencer Banzhaf, Lala Ma, and Christopher Timmins. Environmental Justice: The Economics of Race, Place, and Pollution. Journal of Economic Perspectives, 33(1):185-208, February 2019. 

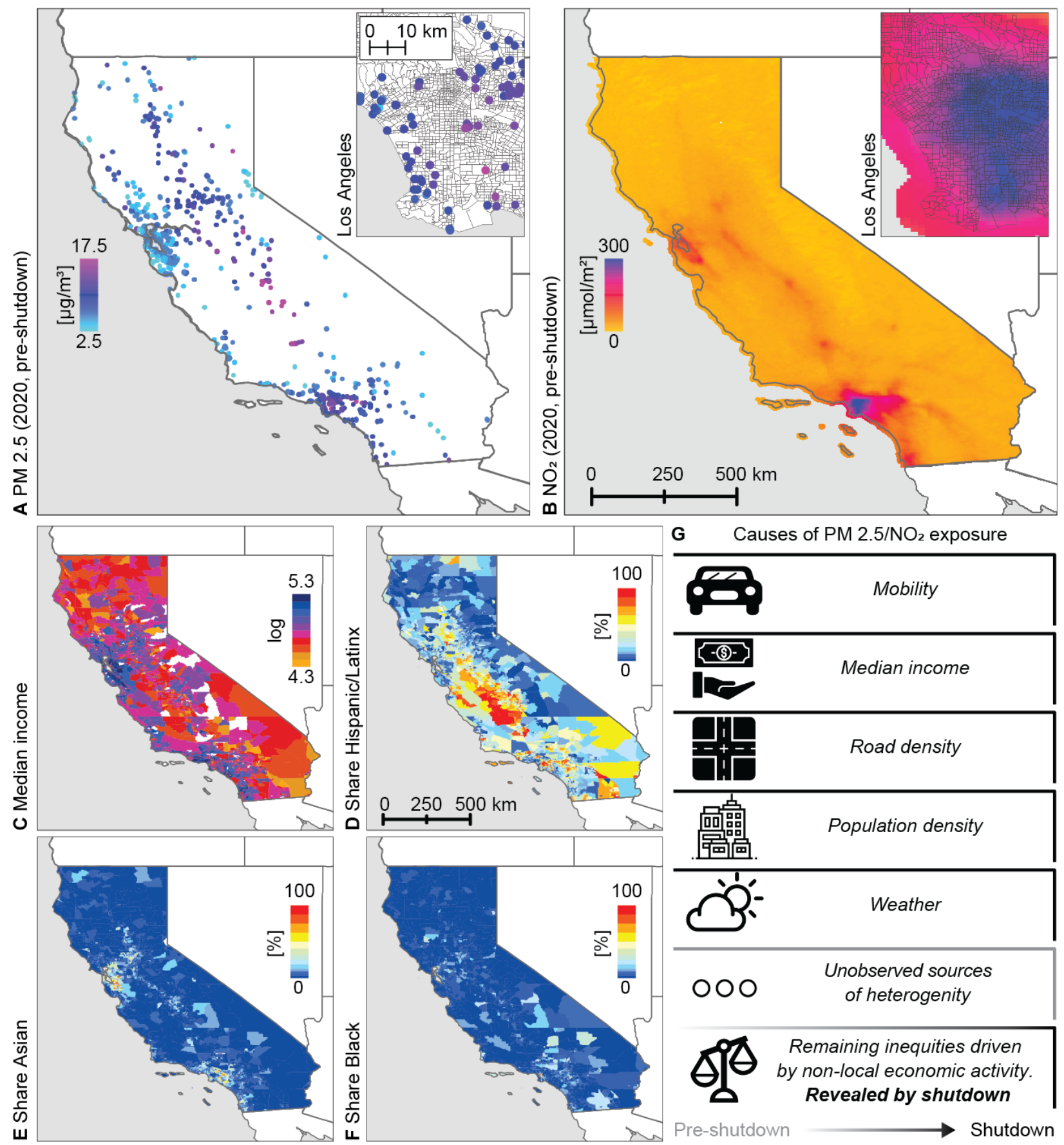

G Causes of $\mathrm{PM} 2.5 / \mathrm{NO}_{2}$ exposure
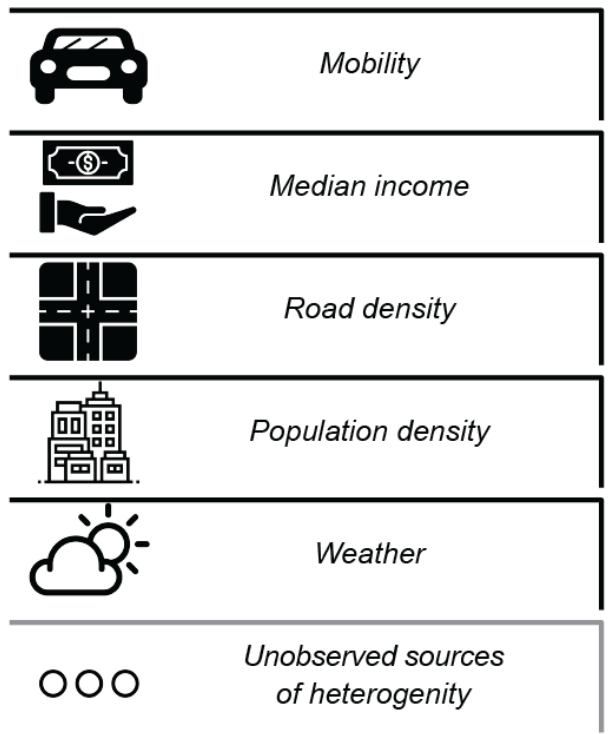

Figure 1: Pollution and demographic data used in this study. A,B Average surface $\mathrm{PM}_{2.5}$ and tropospheric $\mathrm{NO}_{2}$ concentrations in the pre-shutdown period of 2020 in California, United States. C Median income (\$USD) in each census block group from the U.S. Census Bureau 2018 5-year American Community Survey (ACS). D - F Share of the population in each census block group that is Hispanic/Latinx, Asian, or Black, from the ACS. G Schematic showing the many slower-changing (assumed to be static over shorter periods) and higher-frequency factors that contribute to heterogeneous pollution exposures. (Symbols courtesy of Noun Project: Automobile by Symbolon; Income and Highway by Vectors Point; Urban by Eucalup; weather by asianson.design; List by Richard Kunák; inequality by b farias.) 


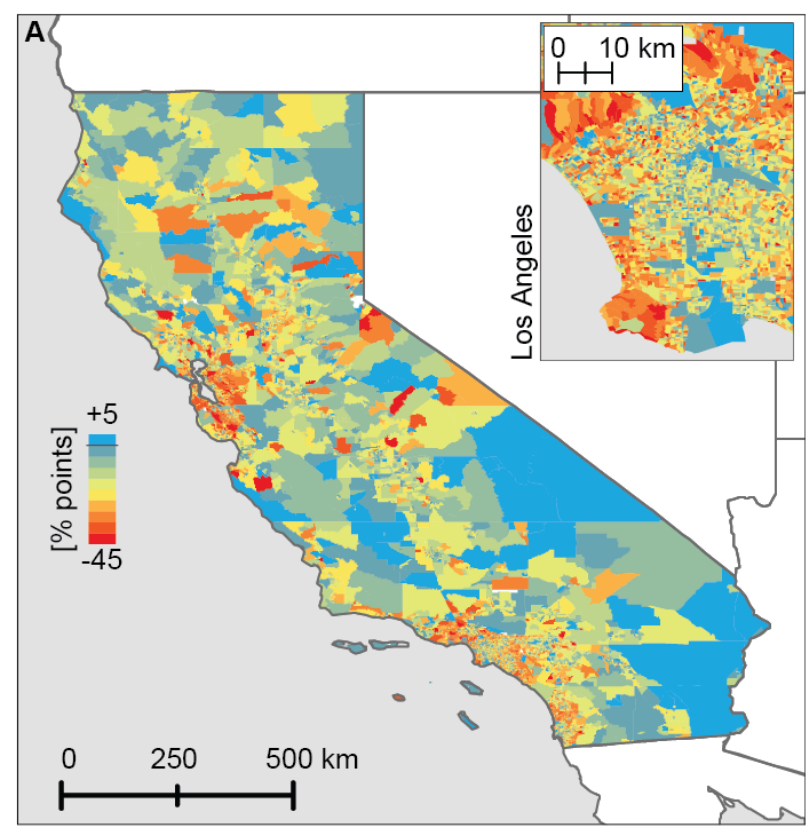

Mobility (shutdown - pre-shutdown)

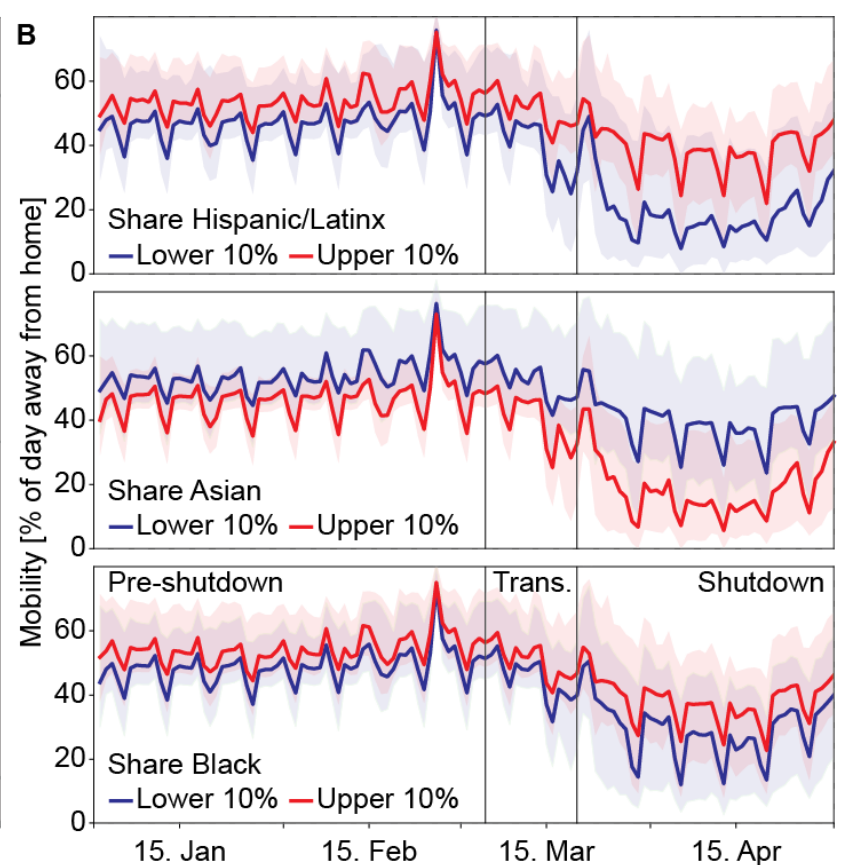

Figure 2: The COVID-19 "Mobility Shock". A Shows the percentage point difference in time spent at home pre-shutdown and during the shutdown at the census block group level in CA, with an inset for the Los Angeles region. B Shows the unequal mobility reductions for the median of the upper and lower 10 percentiles of three different population subsets. Shading indicates the 25 th and 75 th percentiles within each group. Vertical lines indicate the beginning and end of the transition (March 4, 2020 and March 19, 2020) period excluded from our dynamic analysis. 

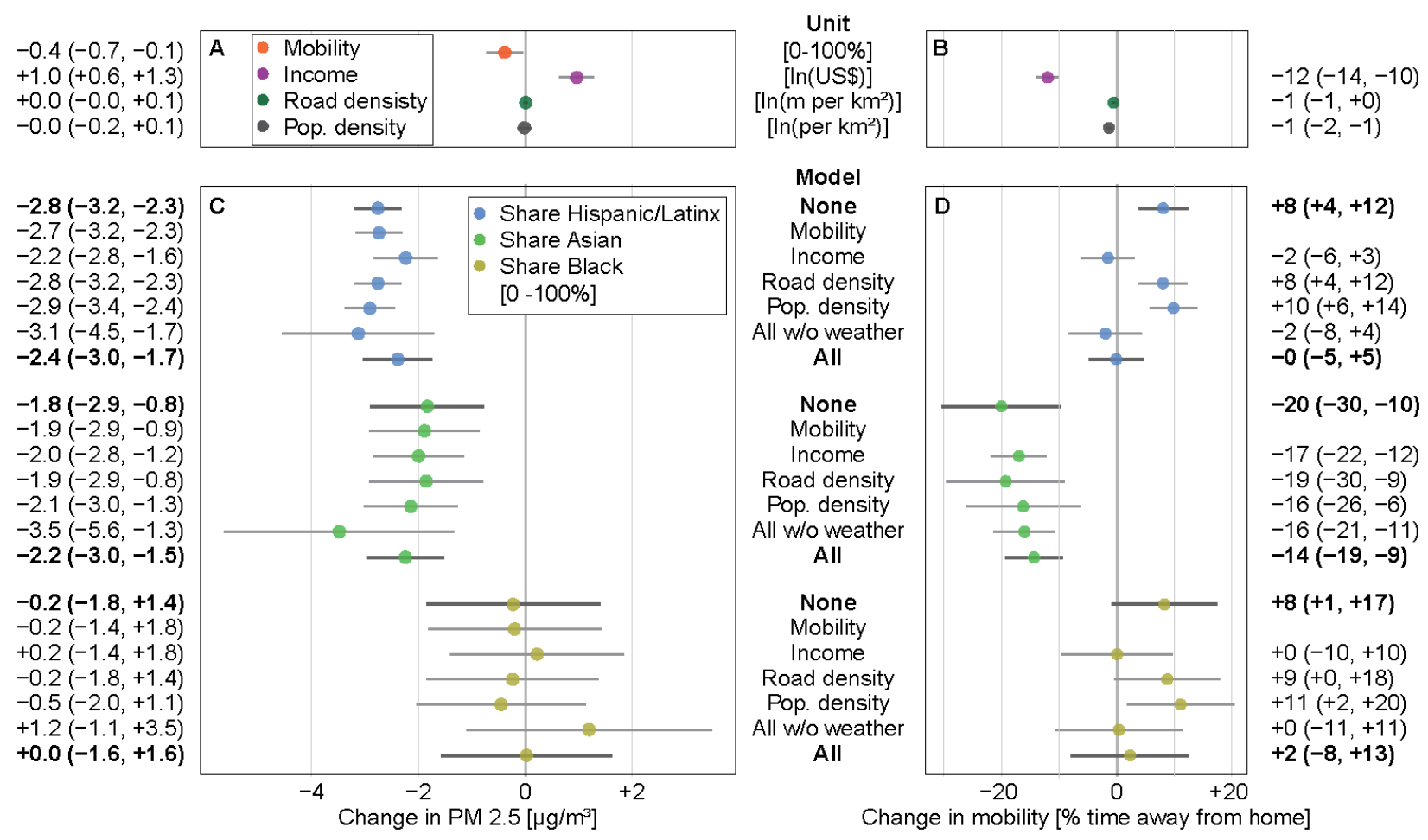

Figure 3: Impact of the economic slowdown on (left) $\mathbf{P M}_{2.5}$ concentrations and (right) mobility. Points show heterogeneous changes across census block group demographics estimated from differences-in-differences models, along with $95 \%$ confidence intervals. Intervals that include zero indicate that there was no differential reduction in exposures across the given gradient. A Changes in daily $\mathrm{PM}_{2.5}$ concentration across the shutdown estimated for various socioeconomic variables. The coefficient for mobility is the estimated difference between 0 and $100 \%$ of time spent at home; the coefficients for (ln) income, road, and population density each represent the impact of an approximate doubling for each variable. $\mathbf{C}$ Changes in $\mathrm{PM}_{2.5}$ concentrations over the shutdown period across different racial and ethnic population shares, estimated with different physical and socioeconomic control variables (labels on right). The coefficients correspond to the expected changes between 0 and $100 \%$ population share at the census block group level. $\mathbf{B}$ and $\mathbf{D}$ show similar estimates, but with mobility as the outcome instead of $\mathrm{PM}_{2.5}$. All four panels compare the post-shutdown difference from 2020 to 2019 to the pre-shutdown difference to account for seasonality. Additionally, estimates were weighted to reflect the distribution of incomes, population shares and other location characteristics across all block groups in California, and correct for the endogenous sampling of ground station locations (see Figure S2B, Methods, and Supporting Information.) 
A Share Hispanic/Latinx [0 - 100\%]
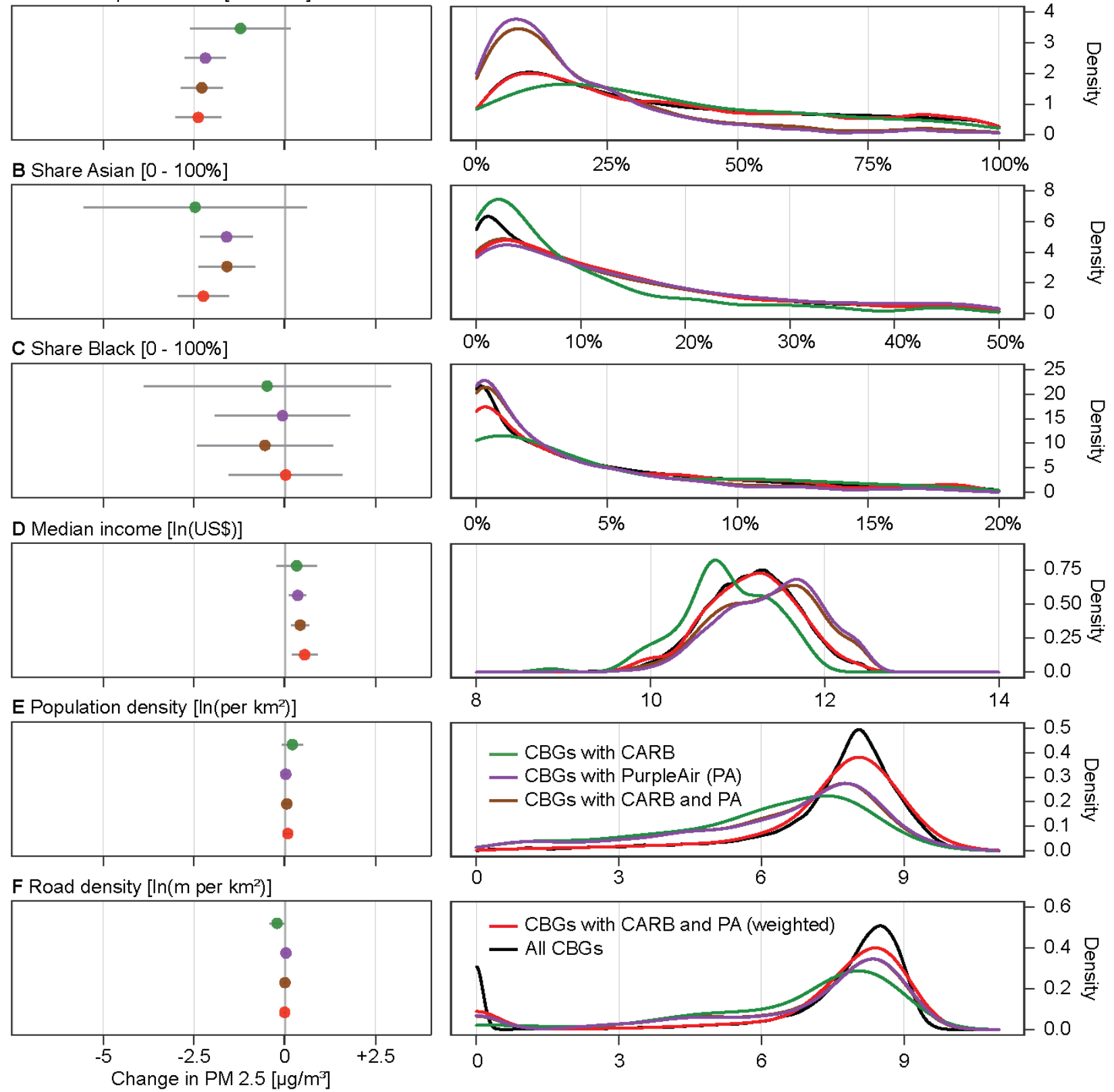

Figure 4: Monitor locations, weighting, and influence on impact estimates. The public (CARB) and private (PurpleAir) $\mathrm{PM}_{2.5}$ sensor networks used in this study are not evenly distributed across the state, which affects how different census block groups contribute to estimated impacts. On the left we show post-shutdown concentration changes across various census block group gradients (as in Figure 3), but estimated using different samples - the public CARB network only (green), the private PurpleAir network only (purple), both together but unweighted (brown), and both together and weighted (red). (These weighted estimates correspond to the estimates presented in Figure 3.) The panels on the right show the representation of demographic and geographic features due to sensor placement by the different sensor networks. Compared to the distribution of these features by all census block groups in California (black lines), the distribution of census block groups with CARB or PurpleAir monitors can be quite different. The distribution of CARB and PurpleAir combined after weighting (red) matches the all-group state-wide distribution much more closely (see Methods). 


\section{Supplemental Information}

This supplement contains:

- Supplemental Text (5 pages)

- Supplemental Figures S1-S10

- Supplemental Tables S1-S3

\section{Supplemental Text}

\section{Data and Methodology Details}

Overview of why the COVID-19 related economic slowdown offers new insight into questions of environmental justice. Figure S1 compares several quantitative approaches to questions of environmental justice present in the literature. Many environmental justice studies note, as in Figure S1A or B, that at any given moment in time (a cross-sectional analysis), ambient pollutant concentrations are higher for communities of color. Here, a best-fit line to cross-sectional observations would lead to an estimate of $\Delta$, or the expected difference in exposure between a $100 \%$ Hispanic/Latinx community and a 100\% non-Hispanic/Latinx community. Accounting for slowermoving confounds in a multi-dimensional analysis, as in $\mathrm{B}$, can change the estimate of $\Delta$. In the case shown, accounting for income can increase the estimate of $\Delta$, if Hispanic/Latinx households tend to have lower incomes than non-Hispanic/Latinx households. Many time-varying factors can also confound this relationship. Importantly, expanding to panel (observations across time) analysis, as in Figure S1C, allows inclusion of weather variables, and various time cycles known to contribute to changes in pollution, like day-of-week and seasonal effects.

While panel studies allow for inclusion of time-varying covariates, it is still the case that the economy (including both point and mobile sources that emit pollutants like primary PM and other precursors that contribute to secondary PM formation), geography (where humans live, including factors like population density and proximity to roads and other steady-state emissions locations), and climate (annual weather cycle and associated daily and seasonal emissions) typically exist together over a fairly narrow set of conditions. Populations change slowly over time, as does the 
general structure of the economy. As such, even in panel analyses, it remains difficult to account for enough factors such that residual exposure disparities can be confidently attributed to the broader scale economy.

A large perturbation to the system, as the COVID-19 pandemic has created, moves one piece of the system (the local and non-local in-person economy) far outside the historical experienced conditions. This allows for a much more robust attribution of the change between pre- and post- slowdown conditions to economic factors. The ability to additionally account for own mobility further allows disaggregation of experienced disparities into those that might be caused by geographic conditions (e.g., communities of color may need to commute more in general, or may be more likely to be essential workers who cannot work from home) and general influence of the broader scale economy. We explain how this intuition also maps to a statistically well-identified question.

Identification of heterogeneous treatment effects. Consider a simplified version of our specifications in the main text with a single interaction of the treatment status (post-shutdown), $d_{i t}$, with a binary, cross-sectional measure of differential exposure to the treatment, $x_{i}$ :

$y_{i t}=\tau d_{i t}+\gamma\left(d_{i t} \times x_{i}\right)+z_{i t}+\mu_{i}+\lambda_{t}+u_{i t}$.

where $z_{i t}$ captures the effects of weather and other observed unit by time variation. All else is defined as before, but note that $y_{i t}$ is now in levels to further simplify the exposition. Using annual differences does not fundamentally alter these results but changes all differences-in-differences (DID) interpretations to a triple DID which allows for more complex forms of unobserved heterogeneity.

This set-up allows us to make two points:

1) With staggered treatment $\left(d_{i t} \neq d_{t}\right.$ for all $\left.i\right)$ and no heterogeneity in the treatment effect $(\gamma=0), \hat{\tau}$ is a standard DID estimate. Differencing over time, rearranging and taking expectation delivers

$\hat{\tau}=\mathbb{E}\left[\Delta y_{i t} \mid \Delta d_{i t}=1\right]-\mathbb{E}\left[\Delta y_{i t} \mid \Delta d_{i t}=0\right]=\left(\tau+\Delta z_{i t}+\Delta \lambda_{t}\right)-\left(\Delta z_{i t}+\Delta \lambda_{t}\right)=\tau$

which can be written as $\Delta \bar{y}_{\text {treat }}-\Delta \bar{y}_{\text {control }}$. However, the overall treatment effect $\tau$ is not statistically identified in our setting, where all observations after a particular calendar date are treated, so that $d_{i t}=d_{t}$ for all $i$, and we do not observe any unit with $\Delta d_{i t}=0$. Hence, $d_{t}$ and $\lambda_{t}$ are perfectly collinear. Omitting the time effects, $\lambda_{t}$ would lead us to be able to estimate $\tau$ but would also lead us to mistakenly attribute state-wide shocks, $\Delta \lambda_{t}$, to the treatment effect.

2) Without staggered treatment but heterogeneity in the treatment effect $(\gamma \neq 0)$, we can only identify the effect of the heterogeneous exposure, $\gamma$, relative to some baseline exposure captured 
by $\tau$. The treatment effect for each observation now becomes $\tau+\gamma x_{i}$ but $\tau$ is still collinear with $\lambda_{t}$. With binary $x_{i}$, the heterogeneous treatment effect is captured by

$$
\begin{aligned}
\hat{\gamma}= & \mathbb{E}\left[\Delta y_{i t} \mid \Delta d_{i t}=1, x_{i}=1\right]-\mathbb{E}\left[\Delta y_{i t} \mid \Delta d_{i t}=1, x_{i}=0\right] \\
& -\left\{\mathbb{E}\left[\Delta y_{i t} \mid \Delta d_{i t}=0, x_{i}=1\right]-\mathbb{E}\left[\Delta y_{i t} \mid \Delta d_{i t}=0, x_{i}=0\right]\right\} \\
= & \mathbb{E}\left[\Delta y_{i t} \mid \Delta d_{i t}=1, x_{i}=1\right]-\mathbb{E}\left[\Delta y_{i t} \mid \Delta d_{i t}=1, x_{i}=0\right] \\
= & \left(\tau+\gamma+z_{i t}+\Delta \lambda_{t}\right)-\left(\tau+z_{i t}+\Delta \lambda_{t}\right)=\gamma
\end{aligned}
$$

which is another DID estimate that compares the effect of the treatment in groups with $x_{i}=1$ to those with $x_{i}=0$. With continuous $x_{i}$ this becomes a generalized DID estimate, where we, for example, compare the effect of the shutdown in block groups with a positive share of the Hispanic/Latinx population to those without any Hispanic/Latinx residents.

Weighting. Ground-based monitor placement is endogenous to several of the variables we interact with the shutdown in order to investigate heterogeneous treatment effects. PurpleAir monitors are privately installed by a non-representative subset of the population which is, among other differences, less racially diverse and lives in higher income neighborhoods than the state-wide average (Figure 4A-F). CARB sensors are often set up to explicitly monitor pollution exposure in vulnerable groups and, hence, tend to be placed in more diverse, less wealthy and more rural block groups (Figure 4A-F). Such choice-based sampling implies that monitor placement is correlated with the error term of our regression equation. Estimation which ignores endogenous sampling is generally biased, but consistent estimates can be obtained by weighting the regression function with the inverse probability of selection (81).

We use iterative proportional fitting - a standard post-stratification procedure - to match the marginal distributions of the endogenous sample of monitors to known census population margins. We determine the marginal distributions of the population by computing the cell frequencies for each of the ACS variables used in the analysis (the Hispanic/Latinx, Asian, and Black population shares, as well as income, road density and population density) per vigintile (20 bins) of the census data. A process called "raking" then finds post-stratification weights which adjust the endogenous sample such that it resembles the set of target distributions (Figure 4A-F). The process is able to fit individual distributions in our data very well, but involves some trade-offs in terms of how well it matches any particular distribution when more than one target variable is used. Calculating the inverse probability of selection directly on a large contingency table would require a much coarser portioning of the data, resulting in a substantially worse fit. All fixed effects regressions in the main text which use ground-based monitors are estimated using weighted least squares if not otherwise noted.

We include a brief investigation into what the weighting tells us about the potential for 
each set of monitors to provide information on disparities. Figure $\mathrm{S} 2 \mathrm{C}$ shows the distribution of weights across CARB and PurpleAir sensor types and shows that more CARB sensors are highly upweighted by our weighting process than PurpleAir sensors. This makes sense-given the endogenous placement of sensors discussed above and in the main text-we should expect some CARB sensors to be deliberately placed where there are few other monitors or in locations where residents would be unlikely to purchase a PurpleAir sensor.

Selection of weather controls We have included variables to control for temperature, relative humidity, and precipitation in the same day $\left(\mathrm{PM}_{2.5}\right)$ or week $\left(\mathrm{NO}_{2}\right)$ as the dependent variable measurement. Most importantly, if correctly specified these controls help reduce the variability caused by weather differences between 2019 and 2020. For example, if areas with larger Asian populations also received more rainfall in 2020 after the shutdown, we might mis-attribute the subsequent reduction in $\mathrm{PM}_{2.5}$ concentrations to the shutdown rather than to the weather. The form of the function which maps these three variables onto concentrations is unknown to us, so we searched for a specification which had good out-of-sample performance, didn't use too many degrees of freedom, and where interacted fixed effects specifications contained few bins with only one observation (effectively dummying out that observation). This exercise sought to find a wellfitting but not overfit specification that left enough observations for the main models to work well, and that did not accidentally remove observations from geographic areas with extremes in one or more of the variables.

Tables S2 and S3 as well as figure S7 show the results of these specification searches. The categories of functional forms that we tried included interacted fixed-width fixed effects (one, two, five, ten, 20) of native units, fixed effects created to split the data into evenly sized groups (decile, vigintile), a cubic spline, polynomial fits (first, second, and third degree), uninteracted fixed effects, and a specification with no adjustment. In the tables, we show each of the first four classes of models using both 2019 and 2020 weather variables or only 2020 weather variables, meaning we included one or both of $f^{2020}(T, R H, P)_{i t}+f^{2019}(T, R H, P)_{i t}$. To test the performance of each specification we ran a regression of weather variables on pollutant using a randomly chosen $70 \%$ of CBGs and tested the performance on the remaining 30\%. Tables S2 and S3 show the average out of sample mean squared error (MSE) for each specification across 100 random splits, the standard deviation of those estimates (sd), the degrees of freedom used by each specification (df), and the number of observations which are dummied out because they singularly correspond to a fixed effect (lost). Both tables are sorted from lowest to highest MSE.

Figure S7 panel B shows the distribution of MSE estimates across 100 70\%/30\% crossvalidations for each specification for $\mathrm{PM}_{2.5}$ and $\mathrm{NO}_{2}$. Panel A shows the coefficients from our main model using a subset of the weather specifications. Our preferred specification is vigintile fixed effects, interacted, for both 2019 and 2020. This specification minimized MSE in the 
$\mathrm{PM}_{2.5}$ regressions and was second in $\mathrm{NO}_{2}$ regressions, while drastically reducing the degrees of freedom used and the observations dummied out. 


\section{Supplemental Figures}
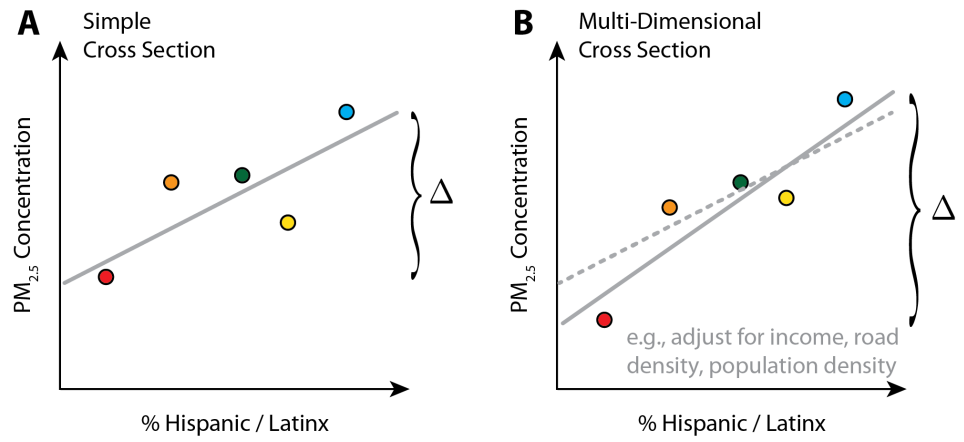

Hispanic / Latinx

$\Delta$ : Predicted difference in $\mathrm{PM}_{2.5}$ for $100 \%$ vs. $0 \%$ Hispanic/Latinx areas.

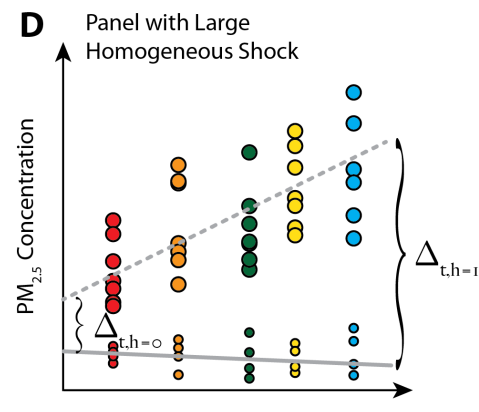

\% Hispanic / Latinx

$\Delta_{\text {thl }=1}-\Delta_{\text {thl=o: }}:$ Predicted difference in change (before/after shutdown) in $\mathrm{PM}_{2,5}$ for $100 \%$ vs. $0 \%$ Hispanic/Latinx areas, accounting for both relatively static factors and time-varying factors.

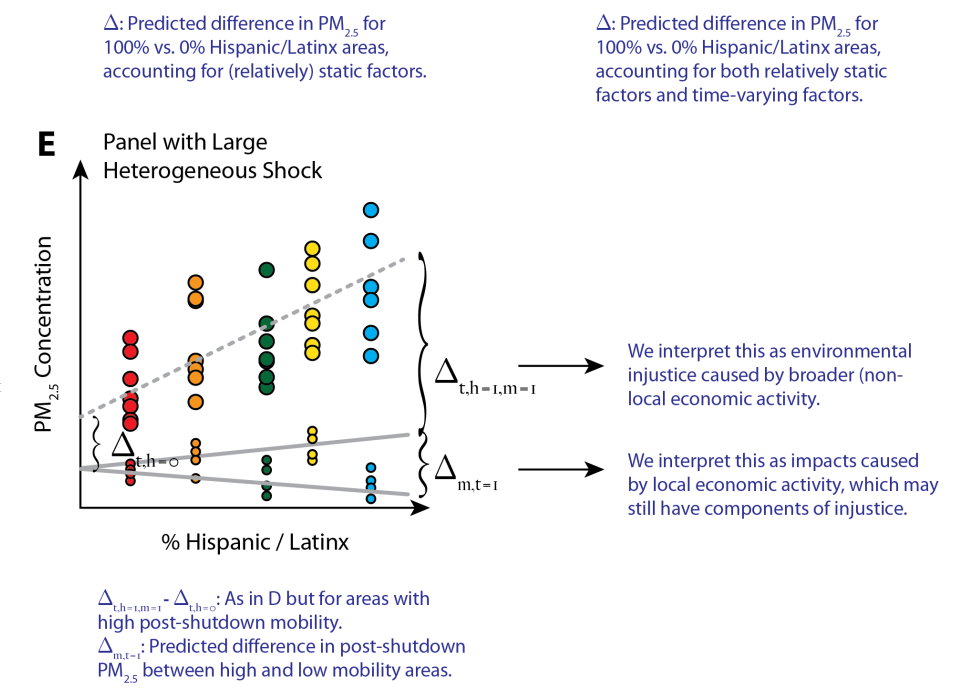

Figure S1: Why the COVID-19 related 'mobility shock' offers new insight into questions of environmental justice. For simplicity, imagine 5 communities across (e.g.) a state, represented by the five colors here. These locations each have a different racial/ethnic composition, represented here for simplicity in one dimension, as the share of the population that is Hispanic/Latinx. Many observations of environmental injustice rely on cross-sectional analyses, either without (A) or accounting for potential slowermoving confounds (B). However, many high-frequency variables contribute to ambient pollution levels and might be correlated with geography and socioeconomic variables. A panel analysis with repeat observations over time $(\mathbf{C})$ allows for inclusion of these types of covariates, and the ability to account for the contribution that (e.g.) natural weather patterns make to exposure disparities. However, even a panel analysis is subject to potential confounding, and interpretation of residual exposure disparities as environmental injustice caused by the economy remains problematic. The COVID-19 economic shock creates a large perturbation that "turns off" a portion of the economy, and thus reveals the footprint of pollution caused by economic activity $(\mathbf{D})$. The ability to account for mobility in this framework (E) further allows the separation of very local activity from broader economic activity (see Supplemental Text). 

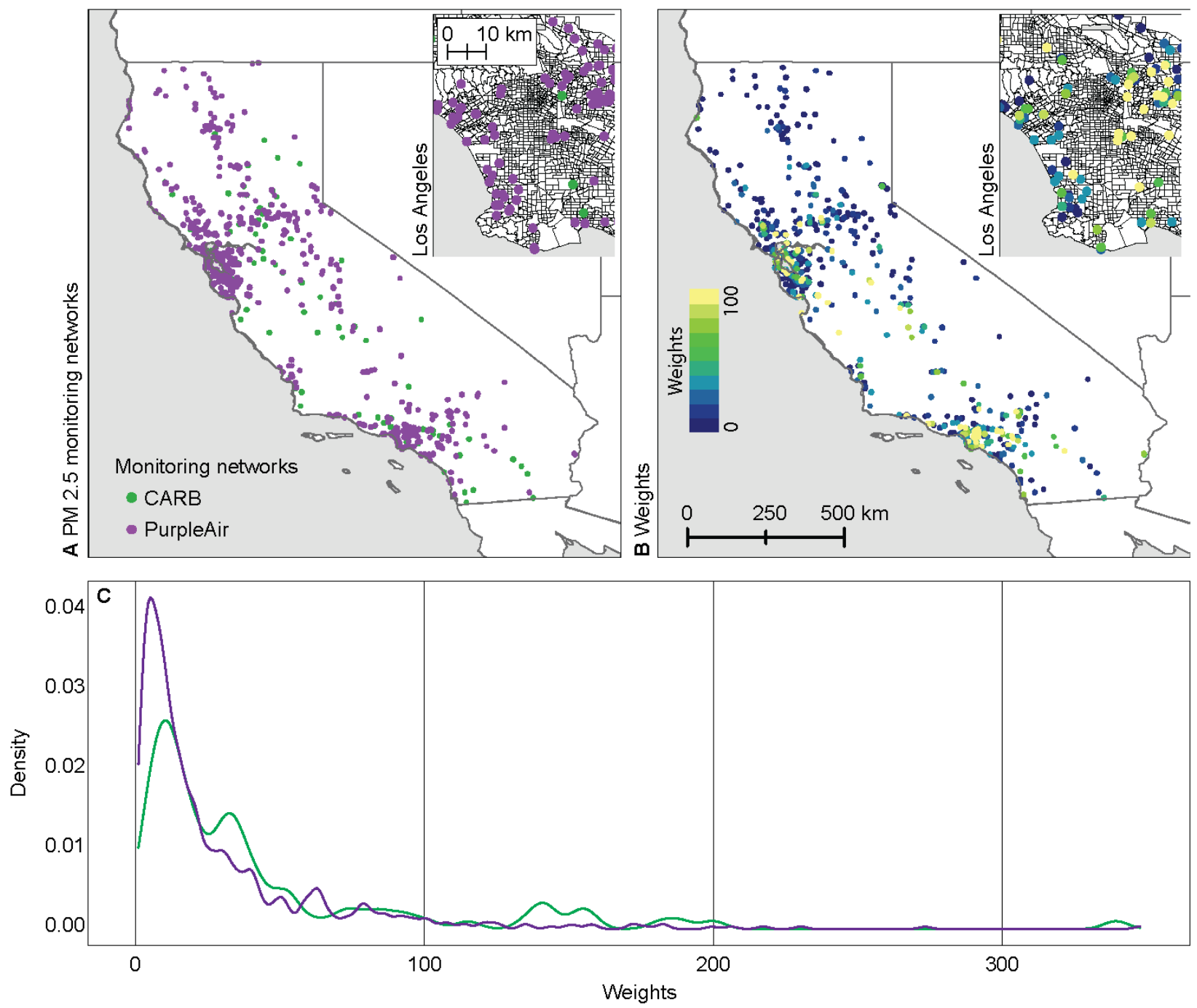

Figure S2: $\mathbf{P M}_{2.5}$ networks and weights. A Location of public (California Air Resources Board (CARB) and United States Environmental Protection Agency (EPA)) $\mathrm{PM}_{2.5}$ monitors, as well as privately-owned PurpleAir $\mathrm{PM}_{2.5}$ monitors used in this study. B Weights used in the model to better represent the Californian population. Each dot represents one census block group. C Distribution of weights generated by the raking process, across sensor types. 

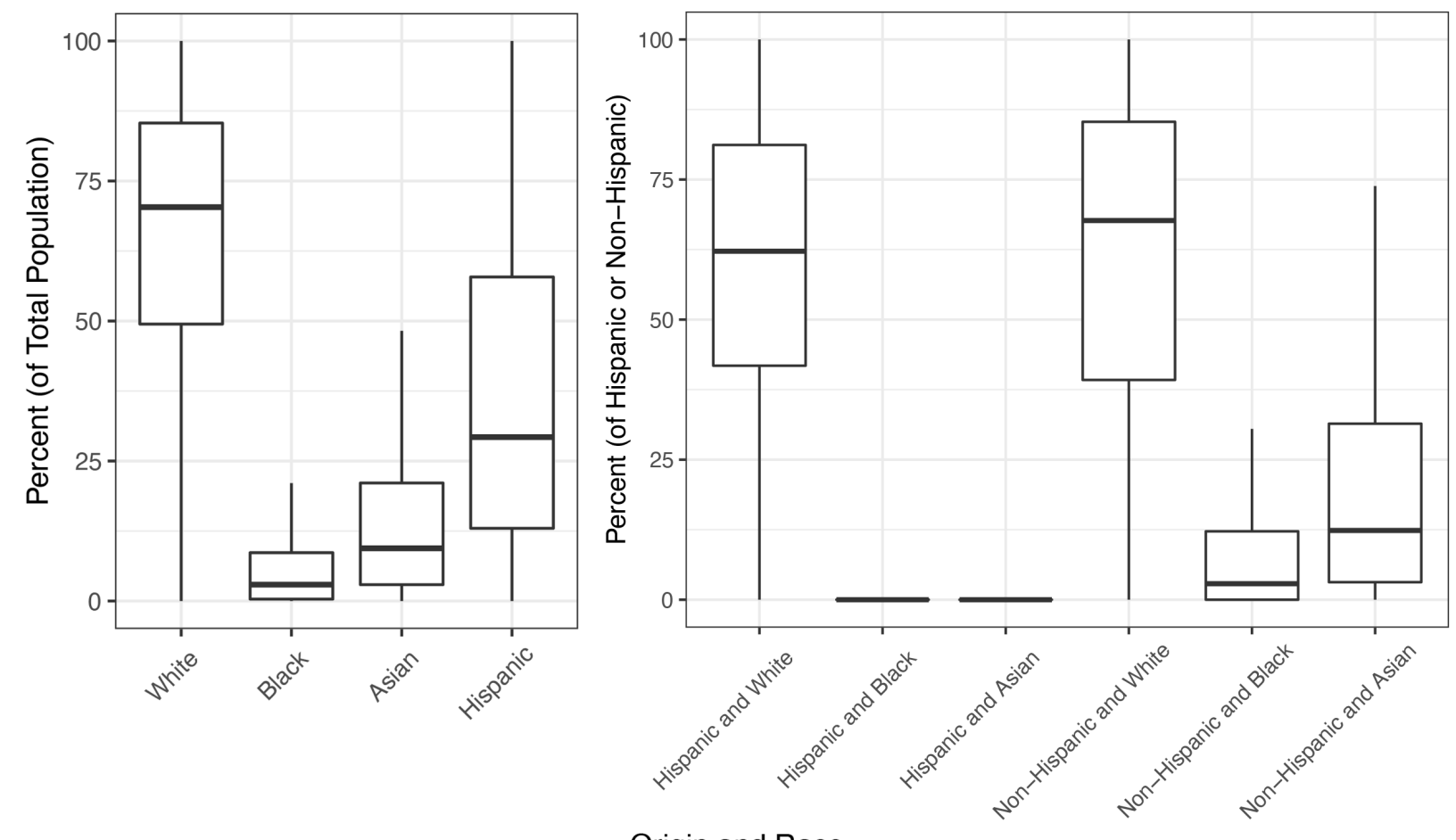

Origin and Race

Figure S3: Summary of origin and race for California census block groups. Distributions of (left panel) the percents of the total population categorized as White, Black, Asian, and Hispanic/Latinx, and (right panel) the percents of either the Hispanic/Latinx or non-Hispanic/Latinx portion of the total population categorized as White, Black, and Asian at the census block group (CBG) level according to the Census Bureau's American Community Survey (ACS). The total number of CBGs was 23,212. Statistical outliers were included in the distribution calculation, but excluded from the visualization. 

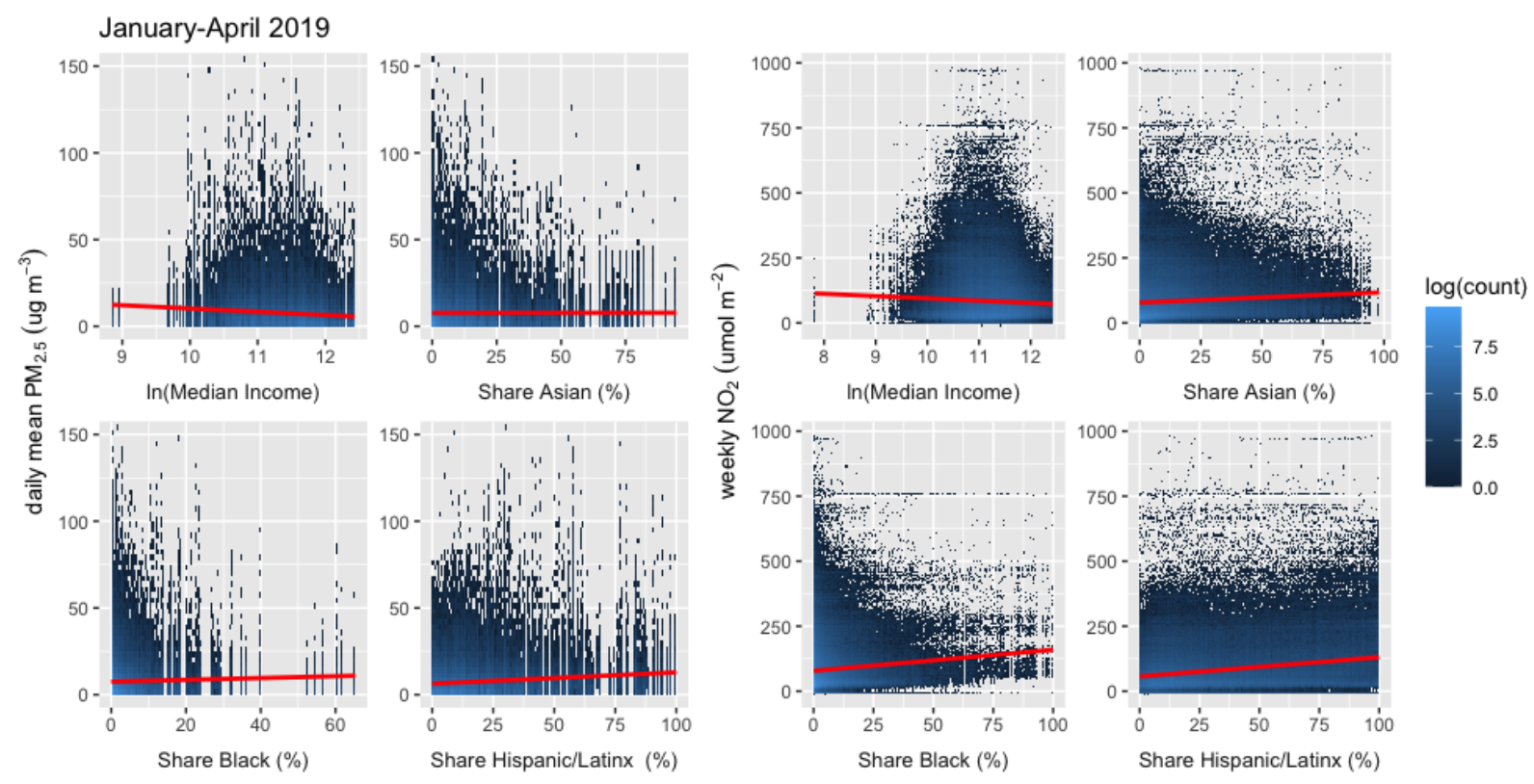

Figure S4: January-April $2019 \mathbf{P M}_{2.5}$ and $\mathrm{NO}_{2}$ with increasing shares of census block group income and racial makeup, without controlling for other sources of heterogeneity. The line represents the best linear fit. 

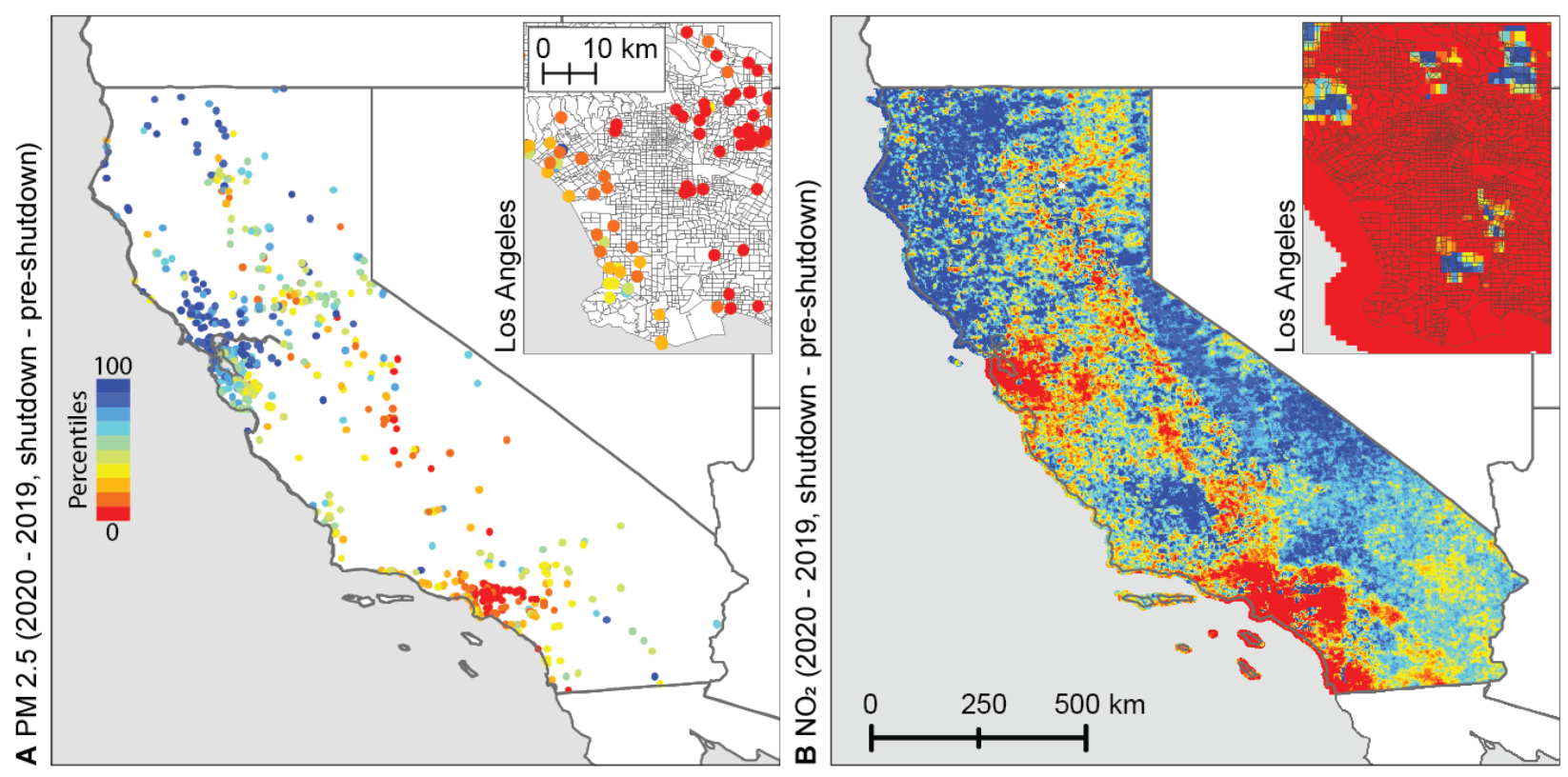

Figure S5: Maps of observed differences-in-differences. A Observed differences (shutdown - pre-shutdown) in the differences (2020 - 2019) in $\mathrm{PM}_{2.5}$. A low percentile/red color indicates a greater reduction in $\mathrm{PM}_{2.5}$. B Same as $\mathrm{A}$ for $\mathrm{NO}_{2}$. 

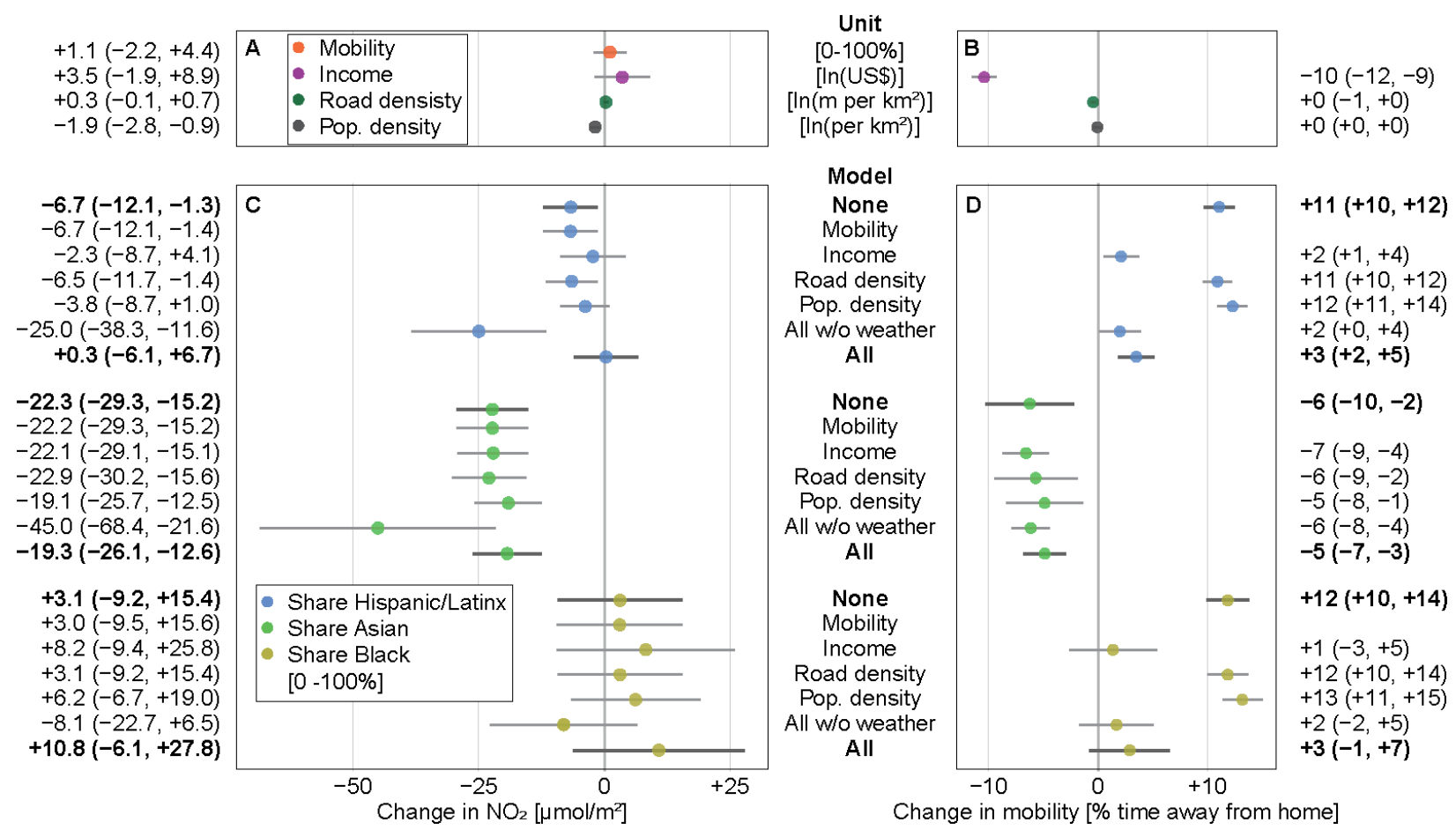

Figure S6: Same as Figure 3, but for weekly $\mathrm{NO}_{2}$ models. Since $\mathrm{NO}_{2}$ measurements are from the TROPOMI satellite instrument, they cover all census block groups in California. Qualitative differences between $\mathrm{NO}_{2}$ estimates $(\mathrm{A}, \mathrm{C})$ and Figure 3A,C thus represent a combination of coverage, and differences in $\mathrm{PM}_{2.5}$ and $\mathrm{NO}_{2}$ distributions. Differences in mobility between these estimates (B,D) and Figure 3B,D are due to coverage differences between the satellite and the ground-based monitoring networks and weekly or rather daily analysis. 


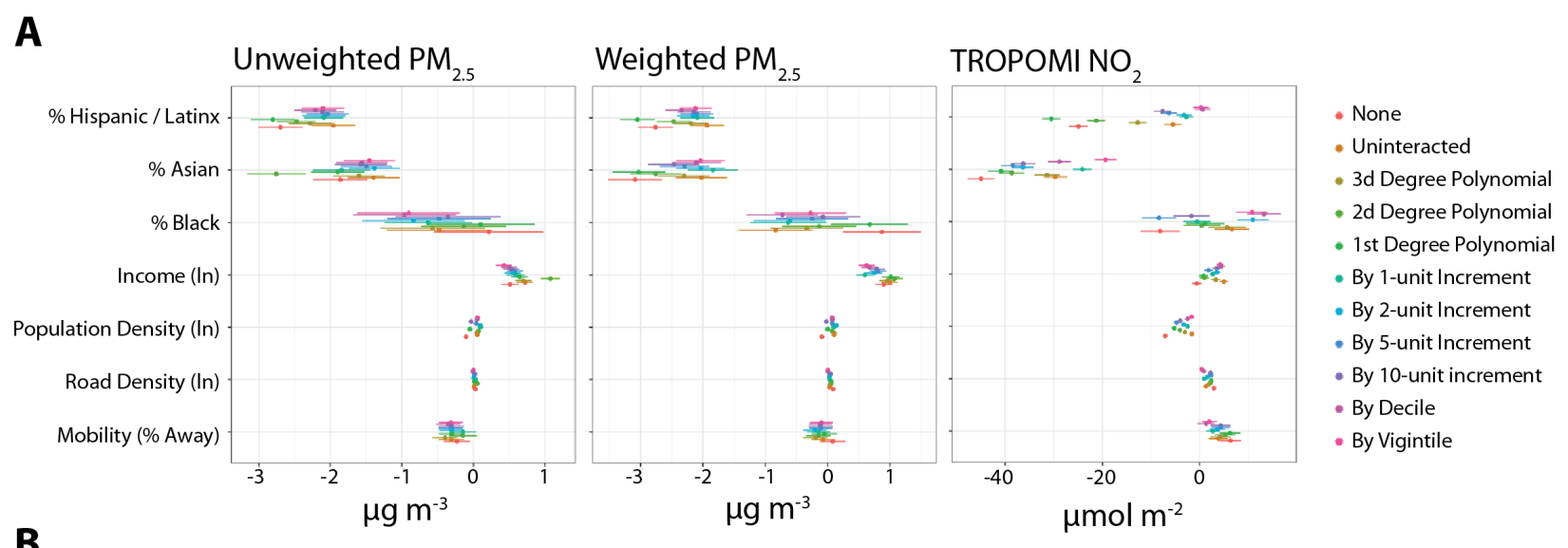

B
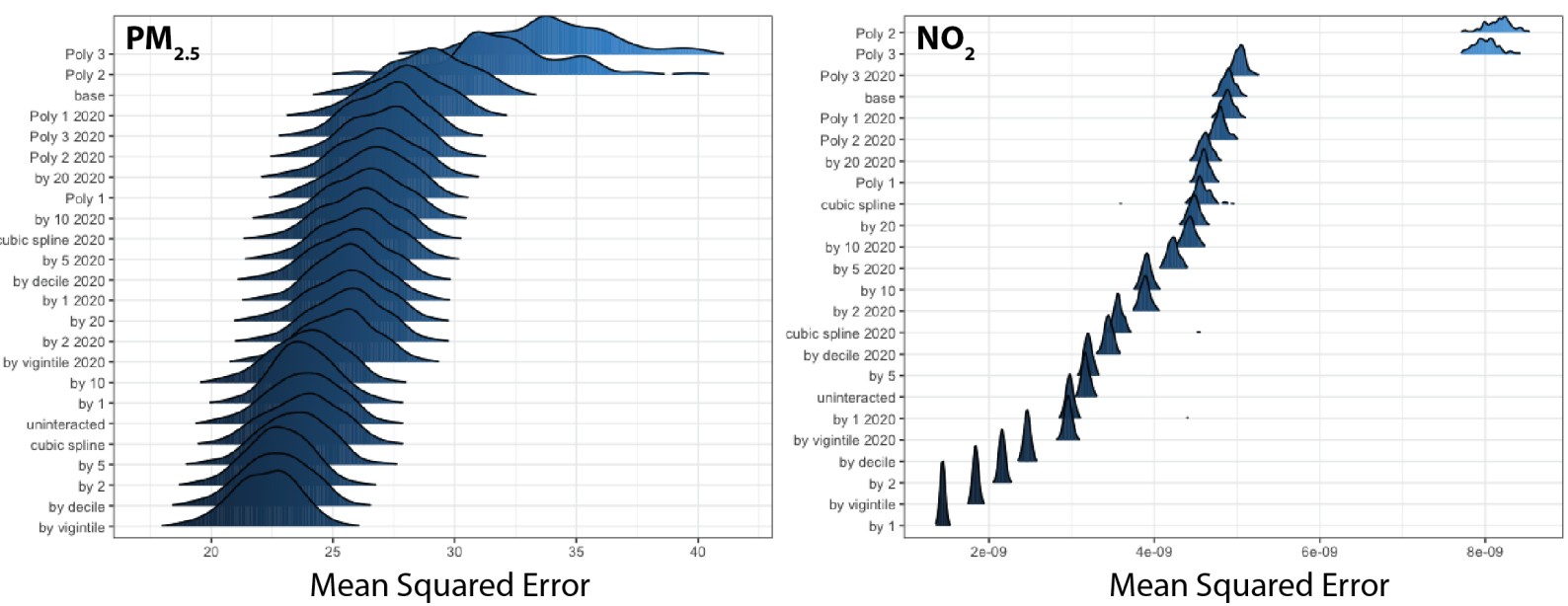

Figure S7: Choice of, and sensitivity to, weather controls specification. A Sensitivity of main results (Figure 3 to different functional forms of climate variables (temperature, precipitation, and relative humidity). B Mean squared error (MSE) of models fit with different functional forms of weather controls and no other covariates, cross-validated, for both $\mathrm{PM}_{2.5}$ and $\mathrm{NO}_{2}$ models (for additional details see the the Selection of weather controls section in the supplement). Tables S3 and S2 show the MSE statistics for each specification, and the number of observations dropped (due to unique weather controls values). We selected interacted vigintile fixed effects as the optimal structure for this analysis based on the combination of lowest MSE for $\mathrm{PM}_{2.5}$ and very few dropped observations, even with full interactions of temperature, precipitation, and relative humidity bins. 


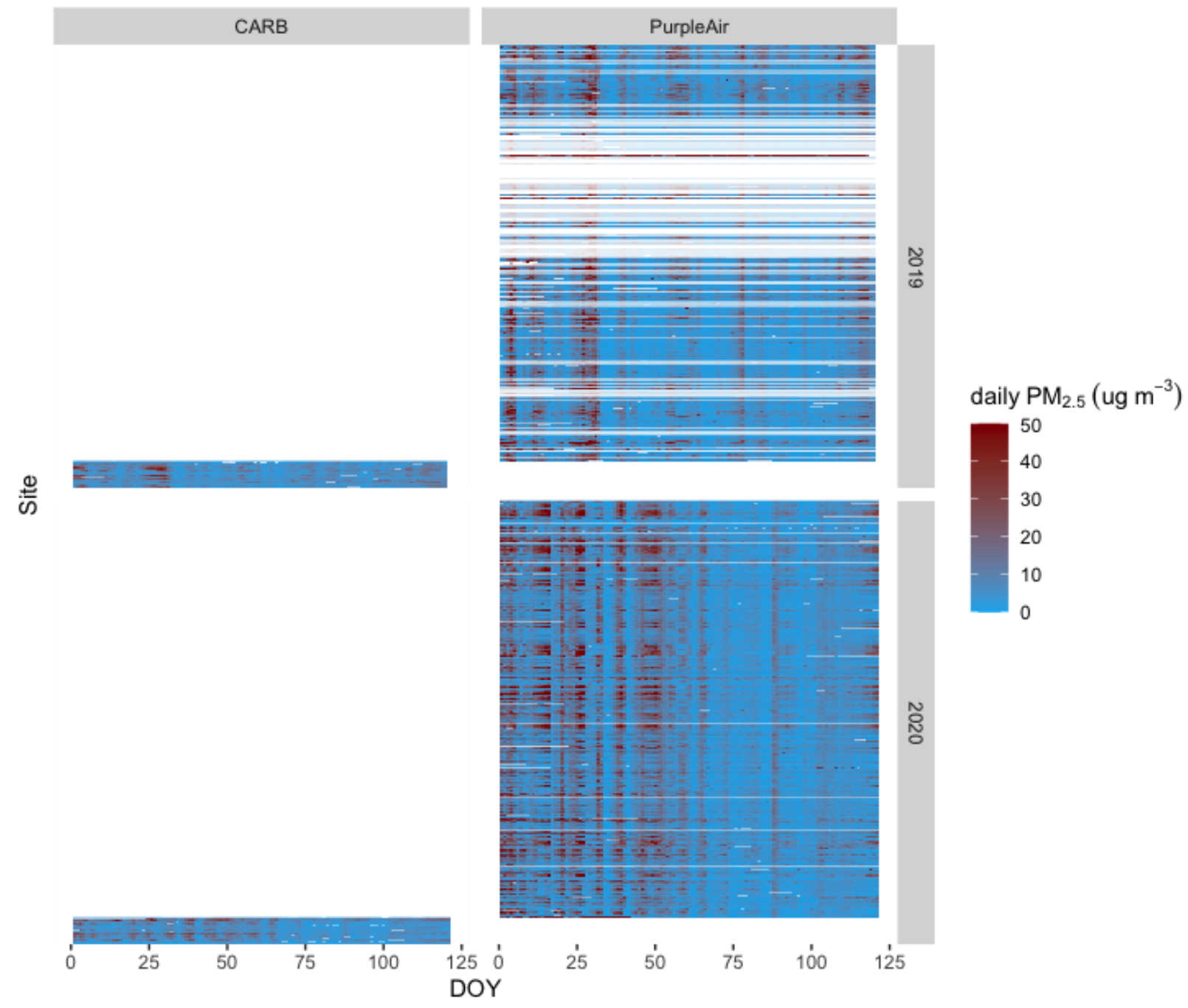

Figure S8: Daily mean PM2.5 measurements from CARB and PurpleAir sensor networks during the study period, in 2019 and 2020, after quality control filtering. 

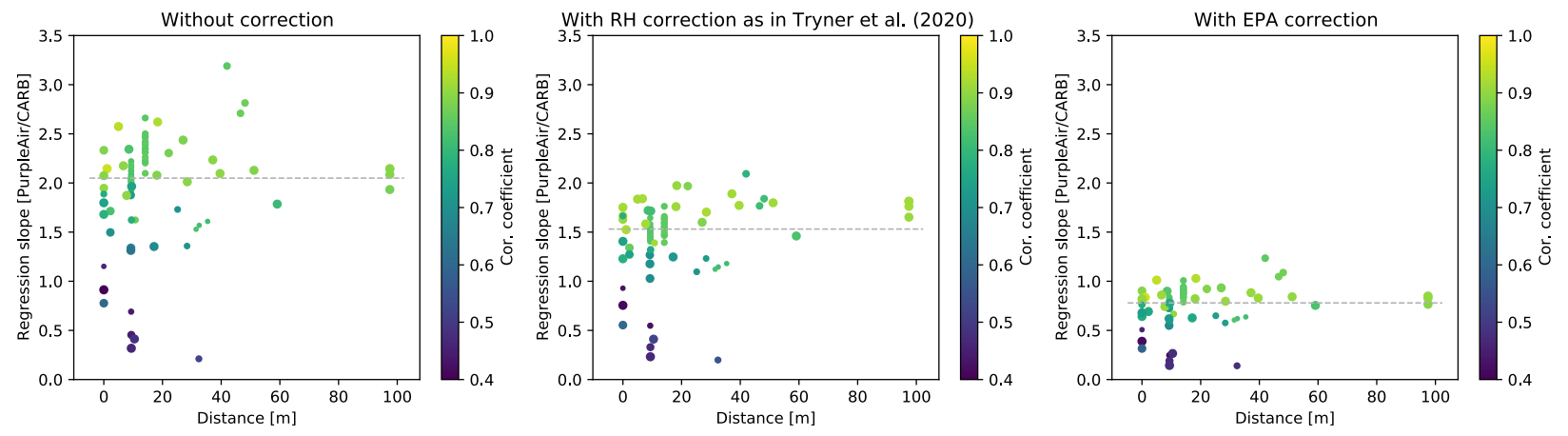

Figure S9: Regression slopes of nearly co-located $(<100 \mathrm{~m})$ PurpleAir and CARB PM2.5 measurements (2019 and 2020 Jan-Apr outdoor in CA) as a function of distance between the PurpleAir and CARB sensors. Color indicates the correlation coefficient. Marker size indicates the number of points used for the regression. The dashed horizontal line indicates the median. Point size indicates number of points available for the sensor pairs. The three panels show the same comparison with no correction (left), the RH correction proposed in Tryner et al. (2020), and the EPA correction used in the study (right). The RH correction may not be sufficient in our case because we use daily mean data. The EPA correction we implement is conservative. Several PurpleAir sensors are paired with the same CARB sensors (in one case as many as 32). 29 unique CARB sensors are represented here, meaning they have a PurpleAir sensor within $100 \mathrm{~m}$. Three sensor pairs were excluded from the figure because the correlation was very low $(<0.2)$. 

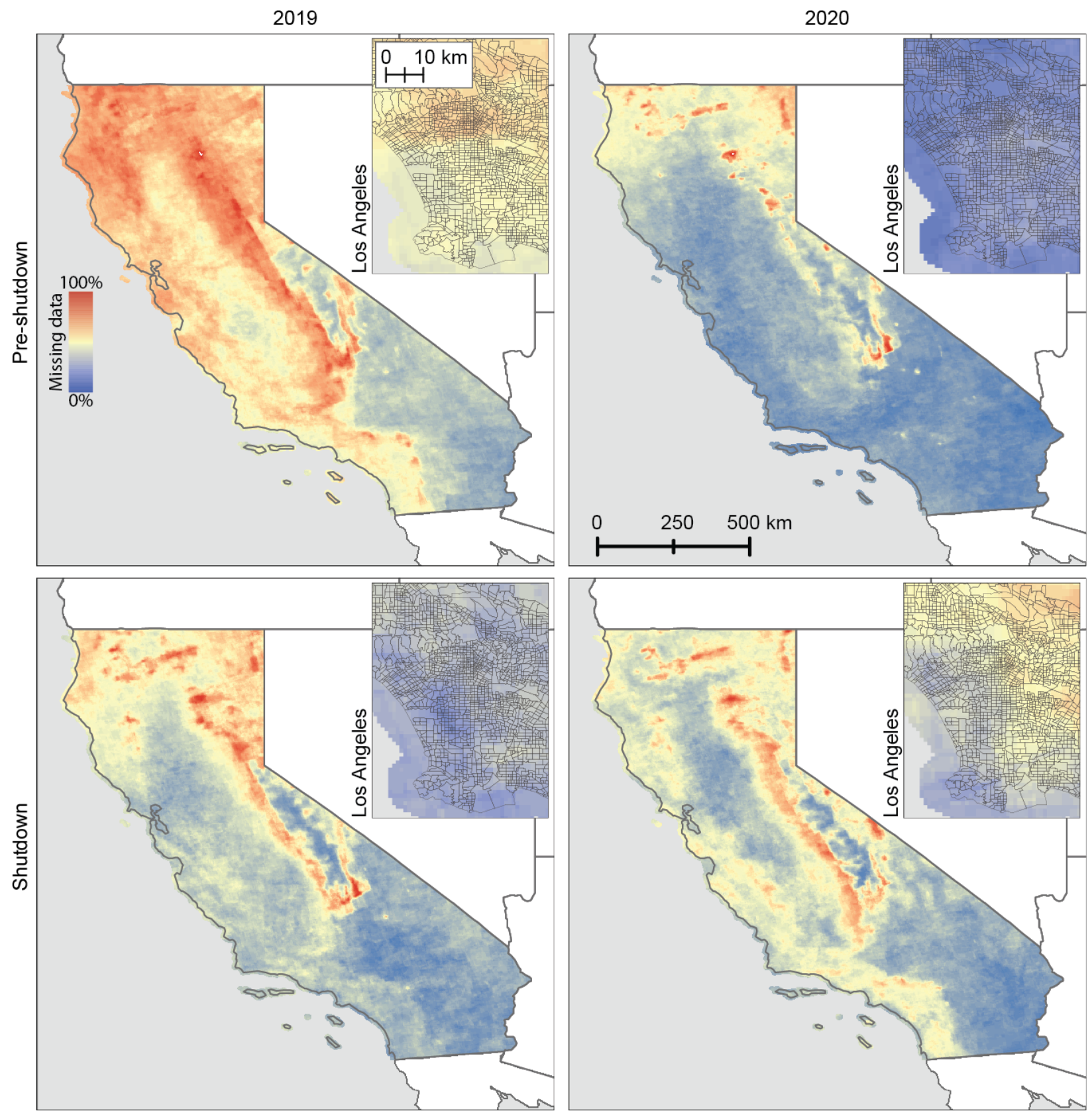

Figure S10: Percentage of Data missing due to low quality or clouds from the TROPOMI $\mathbf{N O}_{2}$ product. For the time periods pre-shutdown and shutdown for 2019 and 2020 the percentage of data missing is shown for each pixel (resolution $1 \mathrm{~km}$ ). 
Supplemental Tables 


\begin{tabular}{|c|c|c|c|c|c|c|}
\hline & \multicolumn{6}{|c|}{ Dependent variable: } \\
\hline & \multicolumn{3}{|c|}{ Difference PM2.5 } & \multicolumn{3}{|c|}{2020 PM2.5 } \\
\hline & (1) & $(2)$ & (3) & $(4)$ & $(5)$ & (6) \\
\hline Mobility & & $\begin{array}{l}-0.217 \\
(0.139)\end{array}$ & $\begin{array}{l}-0.107 \\
(0.158)\end{array}$ & & $\begin{array}{l}-0.221 \\
(0.172)\end{array}$ & $\begin{array}{l}-0.307 \\
(0.191)\end{array}$ \\
\hline$\%$ Hispanic & & & & & & \\
\hline Latinx & $\begin{array}{c}-2.645^{* * *} \\
(0.268)\end{array}$ & $\begin{array}{c}-2.288^{* * *} \\
(0.293)\end{array}$ & $\begin{array}{c}-2.386^{* * *} \\
(0.321)\end{array}$ & $\begin{array}{c}-3.238^{* * *} \\
(0.478)\end{array}$ & $\begin{array}{c}-1.765^{* * *} \\
(0.496)\end{array}$ & $\begin{array}{c}-1.873^{* * *} \\
(0.554)\end{array}$ \\
\hline$\%$ Asian & $\begin{array}{l}-0.949 \\
(0.572)\end{array}$ & $\begin{array}{c}-1.595^{* * *} \\
(0.396)\end{array}$ & $\begin{array}{c}-2.244^{* * *} \\
(0.357)\end{array}$ & $\begin{array}{l}-0.508 \\
(0.865)\end{array}$ & $\begin{array}{l}-0.529 \\
(0.553)\end{array}$ & $\begin{array}{c}-1.436^{* *} \\
(0.580)\end{array}$ \\
\hline$\%$ Black & $\begin{array}{c}-0.639 \\
(0.962)\end{array}$ & $\begin{array}{l}-0.548 \\
(0.955)\end{array}$ & $\begin{array}{c}0.022 \\
(0.799)\end{array}$ & $\begin{array}{l}-2.288 \\
(1.399)\end{array}$ & $\begin{array}{l}-0.615 \\
(1.232)\end{array}$ & $\begin{array}{l}-1.107 \\
(1.426)\end{array}$ \\
\hline Income & & $\begin{array}{c}0.423^{* * *} \\
(0.129)\end{array}$ & $\begin{array}{c}0.544^{* * *} \\
(0.176)\end{array}$ & & $\begin{array}{c}1.069^{* * *} \\
(0.192)\end{array}$ & $\begin{array}{c}1.348^{* * *} \\
(0.294)\end{array}$ \\
\hline Road density & & $\begin{array}{c}0.004 \\
(0.022)\end{array}$ & $\begin{array}{c}-0.004 \\
(0.017)\end{array}$ & & $\begin{array}{c}0.013 \\
(0.033)\end{array}$ & $\begin{array}{l}-0.001 \\
(0.026)\end{array}$ \\
\hline Population density & & $\begin{array}{c}0.052 \\
(0.040)\end{array}$ & $\begin{array}{c}0.081 \\
(0.055)\end{array}$ & & $\begin{array}{c}-0.228^{* * *} \\
(0.049)\end{array}$ & $\begin{array}{c}-0.120^{* *} \\
(0.059)\end{array}$ \\
\hline Weighted & No & No & Yes & No & No & Yes \\
\hline CBG FEs & Yes & Yes & Yes & Yes & Yes & Yes \\
\hline Day FEs & Yes & Yes & Yes & Yes & Yes & Yes \\
\hline $\mathrm{P}(\mathrm{T}, \mathrm{RH}, \mathrm{P}) 2019$ & Yes & Yes & Yes & No & No & No \\
\hline $\mathrm{P}(\mathrm{T}, \mathrm{RH}, \mathrm{P}) 2020$ & Yes & Yes & Yes & Yes & Yes & Yes \\
\hline Observations & 72,953 & 72,953 & 72,953 & 72,953 & 72,953 & 72,953 \\
\hline
\end{tabular}

Table S1: PM2.5 2020-2019 difference vs 2020 values for all of California. Results of three $\mathrm{PM}_{2.5}$ regressions: without controls or weights, with controls and without weights, and with both controls and weights. The first three columns correspond to the estimates in the paper, the second three columns estimate the same quantities using only 2020 data. The dependent variable is the level of $\mathrm{PM}_{2.5}$ rather than the inter-annual difference, and Mobility corresponds to the level of mobility rather than the difference. We only include 2020 weather variables in columns 3-6 rather than the both years. The estimates have slight differences in magnitude but are the same sign and significance level in both specifications. This has two implications. First, the weather controls do a good enough job that differencing out the previous year does not make an enormous difference, though it does add precision to the estimates. Second, it is possible to consistently estimate these effects without access to previous years data. 


\begin{tabular}{rlrrrr}
\hline & model & MSE $(1 \mathrm{e}-11)$ & sd $(1 \mathrm{e}-11)$ & $\mathrm{df}$ & lost \\
\hline 1 & by 1 & 144.0 & 2.2 & 14567 & 4269 \\
2 & by vigintile & 184.0 & 2.7 & 7467 & 612 \\
3 & by 2 & 216.1 & 3.0 & 3870 & 865 \\
4 & by decile & 246.4 & 3.5 & 1350 & 26 \\
5 & by vigintile 2020 & 295.7 & 4.2 & 3659 & 315 \\
6 & by 1 2020 & 299.1 & 14.8 & 5458 & 1453 \\
7 & uninteracted & 317.1 & 4.3 & 317 & 8 \\
8 & by 5 & 320.0 & 4.3 & 519 & 48 \\
9 & by decile 2020 & 344.8 & 4.9 & 668 & 13 \\
10 & cubic spline 2020 & 357.3 & 11.0 & 43 & 0 \\
11 & by 2 2020 & 388.8 & 5.6 & 1310 & 209 \\
12 & by 10 & 390.6 & 5.5 & 107 & 5 \\
13 & by 5 2020 & 422.7 & 6.2 & 168 & 13 \\
14 & by 10 2020 & 442.9 & 6.5 & 34 & 2 \\
15 & by 20 & 447.8 & 6.6 & 29 & 0 \\
16 & cubic spline & 457.3 & 13.9 & 22 & 0 \\
17 & Poly 1 & 460.0 & 6.2 & 7 & 0 \\
18 & by 20 2020 & 461.3 & 7.0 & 11 & 0 \\
19 & Poly 2 2020 & 479.8 & 6.9 & 10 & 0 \\
20 & Poly 1 2020 & 488.3 & 7.6 & 4 & 0 \\
21 & base & 489.5 & 7.6 & 1 & 0 \\
22 & Poly 3 2020 & 503.6 & 6.7 & 19 & 0 \\
23 & Poly 3 & 800.3 & 13.8 & 64 & 0 \\
24 & Poly 2 & 815.8 & 16.1 & 28 & 0 \\
\hline
\end{tabular}

Table S2: NO2 selection of weather controls specification 


\begin{tabular}{|c|c|c|c|c|c|}
\hline & model & MSE & $\mathrm{sd}$ & $\mathrm{df}$ & lost \\
\hline 1 & by vigintile & 22.2 & 1.3 & 4116 & 418 \\
\hline 2 & by decile & 22.6 & 1.3 & 739 & 13 \\
\hline 3 & by 2 & 22.8 & 1.2 & 6111 & 2434 \\
\hline 4 & by 5 & 23.4 & 1.4 & 1114 & 259 \\
\hline 5 & cubic spline & 23.8 & 1.4 & 22 & 0 \\
\hline 6 & uninteracted & 23.8 & 1.4 & 482 & 40 \\
\hline 7 & by 1 & 23.8 & 1.2 & 16477 & 8982 \\
\hline 8 & by 10 & 23.9 & 1.4 & 269 & 34 \\
\hline 9 & by vigintile 2020 & 25.2 & 1.4 & 2040 & 160 \\
\hline 10 & by 22020 & 25.5 & 1.4 & 2181 & 911 \\
\hline 11 & by 20 & 25.6 & 1.5 & 62 & 6 \\
\hline 12 & by 12020 & 25.7 & 1.4 & 6152 & 3674 \\
\hline 13 & by decile 2020 & 25.7 & 1.5 & 362 & 7 \\
\hline 14 & by 52020 & 26.0 & 1.5 & 407 & 91 \\
\hline 15 & cubic spline 2020 & 26.1 & 1.5 & 43 & 0 \\
\hline 16 & by 102020 & 26.3 & 1.5 & 97 & 12 \\
\hline 17 & Poly 1 & 26.6 & 1.4 & 7 & 0 \\
\hline 18 & by 202020 & 26.8 & 1.5 & 25 & 3 \\
\hline 19 & Poly 22020 & 27.0 & 1.5 & 10 & 0 \\
\hline 20 & Poly 32020 & 27.2 & 1.4 & 19 & 0 \\
\hline 21 & Poly 12020 & 27.9 & 1.6 & 4 & 0 \\
\hline 22 & base & 29.0 & 1.6 & 1 & 0 \\
\hline 23 & Poly 2 & 32.1 & 2.4 & 28 & 0 \\
\hline 24 & Poly 3 & 34.1 & 2.4 & 71 & 0 \\
\hline
\end{tabular}

Table S3: PM 2.5 selection of weather controls specification 